TRANSACTIONS OF THE

AMERICAN MATHEMATICAL SOCIETY

Volume 365, Number 7, July 2013, Pages 3729-3751

S 0002-9947(2013)05756-X

Article electronically published on January 24, 2013

\title{
COHOMOLOGICAL UNIQUENESS, MASSEY PRODUCTS AND THE MODULAR ISOMORPHISM PROBLEM FOR 2-GROUPS OF MAXIMAL NILPOTENCY CLASS
}

\author{
ALBERT RUIZ AND ANTONIO VIRUEL
}

\begin{abstract}
Let $G$ be a finite 2-group of maximal nilpotency class, and let $B G$ be its classifying space. We prove that iterated Massey products in $H^{*}\left(B G ; \mathbb{F}_{2}\right)$ do characterize the homotopy type of $B G$ among 2-complete spaces with the same cohomological structure. As a consequence we get an alternative proof of the modular isomorphism problem for 2-groups of maximal nilpotency class.
\end{abstract}

\section{INTRODUCTION}

Let $G$ be a finite $p$-group, and let $B G$ be its classifying space. In this work we consider the cohomological uniqueness of $B G$ : choose some mod- $p$ cohomological invariants and consider $\mathcal{X}$ to be a $p$-complete space such that $B G$ and $\mathcal{X}$ agree on these cohomological invariants; does this mean that $\mathcal{X}$ is homotopy equivalent to $B G$ ? As one may expect, the answer to this question depends on the mod- $p$ cohomological invariants chosen.

In BL97, C. Broto and R. Levi initiated the study of the cohomological uniqueness of $B G$ in terms of Steenrod operations and Bockstein spectral sequences. In this setting, they proved the cohomological uniqueness of the classifying space of every dihedral 2-group [BL97, and every quaternion group BL02. Unfortunately, the available techniques seem not to be strong enough to decide the cohomological uniqueness of the classifying space of semidihedral 2-groups in terms of Steenrod operations and Bockstein spectral sequences.

In order to give a unified approach to the cohomological uniqueness of $B G$, when $G$ is a finite 2-group of maximal nilpotency class (i.e. dihedral, quaternion and semidihedral 2-groups), we propose a different set of mod- $p$ cohomology invariants: the algebra structure of $H^{*}\left(B G ; \mathbb{F}_{p}\right)$ (not taking into account Steenrod operations), and iterated Massey products in this algebra (see Section 2 for precise definitions). In this setting we prove:

Theorem 1.1. Let $G$ be a finite 2-group of maximal nilpotency class. Let $\mathcal{X}$ be a 2-complete topological space having the homotopy type of a $C W$-complex such that $H^{*}\left(\mathcal{X} ; \mathbb{F}_{2}\right) \cong H^{*}\left(B G ; \mathbb{F}_{2}\right)$ as algebras with iterated Massey products. Then $\mathcal{X} \simeq B G$.

Received by the editors April 29, 2011 and, in revised form, November 3, 2011.

2010 Mathematics Subject Classification. Primary 55R35, 20D15.

The first author was partially supported by FEDER-MEC grant MTM2010-20692.

The second author was partially supported by FEDER-MEC grant MTM2010-18089 and Junta de Andalucía grants FQM-213 and P07-FQM-2863.

Both authors were partially supported by Generalitat de Catalunya grant 2009SGR-1092.

(C) 2013 American Mathematical Society 
Proof. In Section 4 we first describe $H^{*}\left(B G ; \mathbb{F}_{2}\right)$ as an algebra with iterated Massey products. This structure is used to construct a homotopy equivalence $\mathcal{X} \rightarrow B G$ whenever $G$ is dihedral (Theorem 5.2), quaternion (Theorem 5.4) or semidihedral (Theorem 5.7).

Besides its own topological interest, the study of the cohomological uniqueness of classifying spaces may have implications in a long-standing algebraic problem: the modular isomorphism problem.

The modular isomorphism problem asks whether a $p$-group $G$ is determined by $\mathbb{F}_{p}[G]$, its group algebra over the field of $p$ elements. That is, given another finite $p$-group $H$ such that $\mathbb{F}_{p}[G] \cong \mathbb{F}_{p}[H]$ as rings, does this mean that $G \cong H$ as groups? The general question still remains open; a positive answer to this question is known for a few families of $p$-groups (see [BK07, Introduction] for an up to date list of results).

Then the interplay between cohomological uniqueness of classifying spaces and the modular isomorphism problem is clear: let $G$ be a finite $p$-group such that $B G$ is cohomology unique when considered a set of mod- $p$ cohomological invariants that can be deduced from the group algebra $\mathbb{F}_{p}[G]$; then the modular isomorphism problem has a positive answer for $G$.

So we obtain a positive answer to the modular isomorphism problem for finite 2-groups of maximal nilpotency class (see Car77, and Bag92 for other approaches to this result):

Corollary 1.2. Let $G$ be a finite 2-group of maximal nilpotency class, and let $H$ be a finite 2 -group such that $\mathbb{F}_{2}[G] \cong \mathbb{F}_{2}[H]$ as rings. Then $G \cong H$ as groups.

Proof. Given any finite $p$-group $K$, the algebra structure with iterated Massey products of $H^{*}\left(B K ; \mathbb{F}_{p}\right)$ can be obtained from the ring structure of $\mathbb{F}_{p}[G]$ by means of the Yoneda cocomplex (e.g., see Bor04, Theorem 2.3 and Lemma 2.4]). Hence, if $\mathbb{F}_{2}[G] \cong \mathbb{F}_{2}[H]$ as rings, then $H^{*}\left(B H ; \mathbb{F}_{2}\right) \cong H^{*}\left(B G ; \mathbb{F}_{2}\right)$ as algebras with iterated Massey products. Then, according to Theorem 1.1, $B G \simeq B H$ (recall that the classifying space of a finite $p$-group is a $p$-complete space), and $G \cong H$ as groups.

We finish this introduction by recalling that iterated Massey products of degree one classes have been previously used by I.C. Borge in Bor04] and Bor01 to provide a cohomological classification of finite $p$-groups. Unfortunately, her results cannot be applied in our topological framework: in Remark 5.5 we give an example showing that iterated Massey products of degree one classes cannot isolate the homotopy type of the classifying space of quaternion groups.

Organization of the paper. In Section 2, we briefly review the definition, main properties and tools needed in the computation of iterated Massey products. Section 3 is devoted to listing and giving some properties of maximal nilpotency class 2 -groups. In Section 4, we compute iterated Massey products in the cohomology of these groups and, in Section 5, we use this structure to prove Theorem 1.1 case by case. There are also two appendices where we develop some explicit computations needed in Section 4: Appendix A provides us with explicit representations of maximal nipotency class 2-groups in $\mathrm{GL}_{m}\left(\mathbb{F}_{2}\right)$ for minimal $m$, and Appendix $\mathrm{B}$ is devoted to expressing the generators in the cohomology of quaternion and semidihedral groups as cochain morphisms in the Yoneda cocomplex. 
Notation. In general the following notation will be used in the rest of the paper: group elements are denoted with lower case letters $(x, y, \ldots)$, while roman capital letters $(X, Y, \ldots)$ and calligraphic capital letters $(\mathcal{X}$ and $\mathcal{Y})$ are used to denote cohomology generators and topological spaces respectively.

Unless otherwise stated, cohomology means cohomology with trivial coefficients over the field $\mathbb{F}_{2}$, so $H^{*}(\mathcal{X})=H^{*}\left(\mathcal{X} ; \mathbb{F}_{2}\right)$. This also implies that when we talk about 2 -complete spaces we are considering the 2-completion in the sense of A.K. Bousfield and D. Kan BK72.

\section{Iterated Massey products: Definition And properties}

In this section we quickly review the theory of iterated Massey products. A more detailed description can be found in [Kra66] or May69.

Let $R$ be a ring with unit. Consider $C^{*}(G, R)$ to be the cochain algebra of the group $G$ with coefficients in $R$, and $d$ the coboundary operator in $C^{*}(G, R)$.

Definition 2.1. Let $\left\{X_{i}\right\}_{1 \leq i \leq n}$ be homogeneous elements in $H^{*}(G, R)$. A defining system for the $n$-fold iterated Massey product $\left\langle X_{1}, \ldots, X_{n}\right\rangle$, is an upper triangular matrix

$$
M=\left\{m_{i, j} \mid 1 \leq i \leq n+1, i<j \leq n+1,(i, j) \neq(1, n+1)\right\}
$$

with coefficients in $C^{*}(G, R)$ such that:

(i) $m_{i, i+1}$ is a representative for $X_{i}$ and

(ii) $d m_{i, j}=\sum_{k=i+1}^{j-1} m_{i, k} \cup m_{k, j}(j \neq i+1)$.

Definition 2.2. Given $M$ a defining system for $\left\langle X_{1}, \ldots, X_{n}\right\rangle$, the value of the product relative to this defining system, denoted $\left\langle X_{1}, \ldots, X_{n}\right\rangle_{M}$, is the element in $H^{*}(G ; R)$ represented by the cocycle:

$$
\sum_{k=2}^{n} m_{1, k} \cup m_{k, n+1} .
$$

The $n$-fold iterated Massey product $\left\langle X_{1}, \ldots, X_{n}\right\rangle$ is defined as the set of elements which can be written as $\left\langle X_{1}, \ldots, X_{n}\right\rangle_{M}$ for some defining system $M$. The indeterminacy in the iterated Massey product $\left\langle X_{1}, \ldots, X_{n}\right\rangle$ is defined as the set of elements $Z$ which can be expressed as $Z=Y_{1}-Y_{2}$ for $Y_{1}$ and $Y_{2}$ in $\left\langle X_{1}, \ldots, X_{n}\right\rangle$.

We now enumerate some of the properties which are used later:

(i) The iterated Massey product $\left\langle X_{1}, \ldots, X_{n}\right\rangle$ is not defined for all $X_{1}, \ldots, X_{n}$ in $H^{*}(G ; R)$. For example, $\left\langle X_{1}, X_{2}, X_{3}\right\rangle$ is defined if and only if $X_{1} \cup X_{2}=$ $X_{2} \cup X_{3}=0$.

(ii) The degree of an element in $\left\langle X_{1}, \ldots, X_{n}\right\rangle$ is $\sum \operatorname{deg}\left(X_{i}\right)-n+2$.

(iii) If $f: \mathcal{X} \rightarrow \mathcal{Y}$ is a continuous map of topological spaces, and $Y_{1}, \ldots, Y_{r}$ are cohomology classes in $H^{*}(\mathcal{Y} ; R)$ such that $\left\langle Y_{1}, \ldots, Y_{r}\right\rangle$ is defined, then so is $\left\langle f^{*}\left(Y_{1}\right), \ldots, f^{*}\left(Y_{r}\right)\right\rangle$ and

$$
f^{*}\left(\left\langle Y_{1}, \ldots, Y_{r}\right\rangle\right) \subset\left\langle f^{*}\left(Y_{1}\right), \ldots, f^{*}\left(Y_{r}\right)\right\rangle .
$$

Moreover, if $f^{*}$ is an isomorphism, equality holds. 
The next result follows from May's proof of May69, Theorem 1.5]:

Lemma 2.3. Let $f: \mathcal{X} \rightarrow \mathcal{Y}$ be a continuous map such that $f^{k}: H^{k}(\mathcal{Y} ; R) \rightarrow$ $H^{k}(\mathcal{X} ; R)$ is an isomorphism for $k \leq n$. Let $Y_{1}, \ldots, Y_{r}$ be elements in $H^{*}(\mathcal{Y} ; R)$ such that $\sum \operatorname{deg}\left(Y_{i}\right)-r+2 \leq n$. Then:

(a) $\left\langle Y_{1}, \ldots, Y_{r}\right\rangle$ is defined if and only if $\left\langle f^{*}\left(Y_{1}\right), \ldots, f^{*}\left(Y_{r}\right)\right\rangle$ is so.

(b) $f^{*}\left(\left\langle Y_{1}, \ldots, Y_{r}\right\rangle\right)=\left\langle f^{*}\left(Y_{1}\right), \ldots, f^{*}\left(Y_{r}\right)\right\rangle$.

Let $p$ be a fixed prime. In this work, we compare the mod- $p$ cohomology algebras (with iterated Massey products) of spaces. This is done by considering a special kind of morphism:

Definition 2.4. Let $\varphi: H^{*}\left(\mathcal{Y} ; \mathbb{F}_{p}\right) \rightarrow H^{*}\left(\mathcal{X} ; \mathbb{F}_{p}\right)$ be a morphism (not necessarily induced by a continuous map of topological spaces). We say that $\varphi$ is an $\mathcal{M}$ isomorphism if

(1) $\varphi$ is an $\mathbb{F}_{p}$-algebra isomorphism and

(2) for all $r \geq 1$ and $Y_{1}, \ldots, Y_{r}$ elements in $H^{*}\left(\mathcal{Y} ; \mathbb{F}_{p}\right)$ such that $\left\langle Y_{1}, \ldots, Y_{r}\right\rangle$ is defined, then $\left\langle\varphi\left(Y_{1}\right), \ldots \varphi\left(Y_{r}\right)\right\rangle$ is defined and

$$
\varphi\left(\left\langle Y_{1}, \ldots, Y_{r}\right\rangle\right)=\left\langle\varphi\left(Y_{1}\right), \ldots, \varphi\left(Y_{r}\right)\right\rangle .
$$

Definition 2.5. Let $\mathcal{X}$ and $\mathcal{Y}$ be topological spaces. We say that $\mathcal{X}$ and $\mathcal{Y}$ are $\mathcal{M}$-comparable if there exists an $\mathcal{M}$-isomorphism $\varphi: H^{*}\left(\mathcal{Y} ; \mathbb{F}_{p}\right) \rightarrow H^{*}\left(\mathcal{X} ; \mathbb{F}_{p}\right)$. We say that $\mathcal{X}$ is determined by its $\mathcal{M}$-cohomology if for any $p$-complete space $\mathcal{Y}$ having the homotopy type of a $C W$-complex and such that it is $\mathcal{M}$-comparable to $\mathcal{X}$ we have that $\mathcal{X} \simeq \mathcal{Y}$.

The easiest examples of spaces determined by its $\mathcal{M}$-cohomology are provided by classifying spaces of some particular families of $p$-groups:

Proposition 2.6. Let $E$ be an elementary abelian p-group. Then BE is determined by its $\mathcal{M}$-cohomology.

Proof. Let $E$ be a rank $r$ elementary abelian $p$-group. First we prove the algebra structure of $H^{*}\left(B E ; \mathbb{F}_{p}\right)$ with iterated Massey products determines the Steenrod operations and the Bockstein spectral sequence. We consider two cases:

- If $p=2$, then $H^{*}\left(B E ; \mathbb{F}_{2}\right)=\mathbb{F}_{2}\left[X_{1}, \ldots, X_{r}\right]$, where every $X_{i}$ has degree 1 . Therefore, the unstability axiom forces $\mathrm{Sq}^{1} X_{i}=X_{i}^{2}$, and this determines completely the Steenrod operations and the Bockstein spectral sequence.

- If $p>2$, then $H^{*}\left(B E ; \mathbb{F}_{2}\right)=\Lambda\left(U_{1}, \ldots, U_{r}\right) \otimes \mathbb{F}_{2}\left[V_{1}, \ldots, V_{r}\right]$, where every $U_{i}$ has degree 1 , and every $V_{i}$ has degree 2 . Again, the unstability axiom forces $\mathcal{P}^{1} U_{i}=0$ and $\mathcal{P}^{1} V_{i}=V_{i}^{p}$, and this determines completely the Steenrod operations. Moreover, according to [Kra66, Theorem 19], the $p$-fold iterated Massey product $\left\langle U_{i}, U_{i}, \ldots, U_{i}\right\rangle$ equals $\beta_{1}\left(U_{i}\right)=V_{i}$, where $\beta_{1}$ is the primary Bockstein operator. So, in this case, Massey products completely determine the Bockstein spectral sequence.

Finally, $B E$ is determined by its cohomology with Steenrod operations and the Bockstein spectral sequence [BL97, Proposition 1.5]; thus the result follows.

2.1. Iterated Massey products of degree one elements. In this subsection we recall the work of W.G. Dwyer Dwy75, Section 2], which relates iterated Massey products of degree one elements in the cohomology of a group and representations of this group in the upper triangular matrices. 
Let $U(R, n)$ be the multiplicative group of all upper triangular $n \times n$ matrices over $R$ which agree with the identity matrix along the diagonal. The subgroup $Z(R, n)$ of $U(R, n)$ consists of matrices which are identically zero except along the diagonal and at position $(1, n)$. We get that $Z(R, n) \cong R$ and it is contained in the center of $U(R, n)$, so it gives rise to the central extension:

$$
Z(R, n) \rightarrow U(R, n) \rightarrow \bar{U}(R, n) \stackrel{\text { def }}{=} U(R, n) / Z(R, n) .
$$

Given a group homomorphism $\phi: G \rightarrow U(R, n)$, the image of $g \in G$ is a matrix with coefficients $\phi_{i, j}(g) \in R$. Note that the elements $\phi_{i, i+1}(g)$ satisfy the equation

$$
\phi_{i, i+1}\left(g_{1} g_{2}\right)=\phi_{i, i+1}\left(g_{1}\right)+\phi_{i, i+1}\left(g_{2}\right) .
$$

So $\phi_{i, i+1}$ are group morphisms from $G$ to $R$, and thus represent cohomology classes in $H^{1}(G ; R)$. These elements are called near diagonal elements of $\phi$.

Theorem 2.7 (Dwy75). Let $X_{1}, \ldots, X_{n}$ be elements in $H^{1}(G ; R)$. There is a one-one correspondence $M \leftrightarrow \phi_{M}$ between defining systems $M$ for $\left\langle X_{1}, \ldots, X_{n}\right\rangle$ and group homomorphisms $\phi_{M}: G \rightarrow \bar{U}(R, n+1)$ which have $-X_{1}, \ldots,-X_{n}$ as near-diagonal components. Moreover, given $\phi_{M}$, we can pull back the extension in equation (11), getting an extension of $G$ by $R$. Then $\left\langle X_{1}, \ldots, X_{n}\right\rangle_{M}$ is exactly the characteristic class of this extension.

2.2. Iterated Massey products in higher degrees. In order to compute iterated Massey products of higher degree elements we use the Yoneda cocomplex [Bor01, Section 2.5]:

Fix $G$ a $p$-group and $P_{\bullet} \rightarrow \mathbb{F}_{p}$ a $\mathbb{F}_{p}[G]$-free resolution with differentials $\partial_{i}: P_{i+1} \rightarrow$ $P_{i}$.

Definition 2.8. The Yoneda cocomplex $\operatorname{Hom}_{\mathbb{F}_{p}[G]}^{(\bullet)}\left(P_{\bullet}, P_{\bullet}\right)$ is defined as:

- In degree $i$ we have the $\mathbb{F}_{p}[G]$-module:

$$
\operatorname{Hom}_{\mathbb{F}_{p}[G]}^{i}\left(P_{\bullet}, P_{\bullet}\right)=\prod_{n \in \mathbb{Z}} \operatorname{Hom}_{\mathbb{F}_{p}[G]}\left(P_{n+i}, P_{n}\right), \quad i \geq 0
$$

and 0 otherwise.

- The differential of $\phi^{i}=\left\{\phi_{n}^{i}: P_{n+i} \rightarrow P_{n}\right\}_{n \in \mathbb{Z}} \in \operatorname{Hom}_{\mathbb{F}_{p}[G]}^{i}\left(P_{\bullet}, P_{\bullet}\right)$, is defined as

$$
\delta^{i}\left(\phi_{n}^{i}\right)=\partial_{n-1} \phi_{n}^{i}-(-1)^{i} \phi_{n-1}^{i} \partial_{n+i-1} .
$$

- The algebra structure in the cohomology of $\operatorname{Hom}_{\mathbb{F}_{p}[G]}^{(\bullet)}\left(P_{\bullet}, P_{\bullet}\right)$ is induced by the composition of elements as a cochain morphism.

The following result tells us that we can use this tool to work with the cohomology of a group:

Theorem 2.9 ([Bor01] $) \cdot H^{i}\left(\operatorname{Hom}_{\mathbb{F}_{p}[G]}^{(\bullet)}\left(P_{\bullet}, P_{\bullet}\right), \delta^{\bullet}\right) \cong H^{i}\left(G ; \mathbb{F}_{p}\right)$ for all $i \geq 0$.

\section{Maximal NiLPotency Class 2-Groups}

The maximal nilpotency class finite 2-groups are precisely the dihedral, quaternion and semidihedral groups. 
Consider the following finite presentations of these three families of 2-groups:

$$
\begin{aligned}
& D_{2^{n}}=\left\langle x, y \mid x^{2^{n-1}}=1, y^{2}=1, y x y^{-1}=x^{-1}\right\rangle \\
& Q_{2^{n}}=\left\langle x, z \mid x^{2^{n-1}}=1, z^{2}=x^{2^{n-2}}, z x z^{-1}=x^{-1}\right\rangle \text { and } \\
& S D_{2^{n}}=\left\langle x, t \mid x^{2^{n-1}}=1, t^{2}=1, t x t^{-1}=x^{2^{n-2}-1}\right\rangle .
\end{aligned}
$$

Also consider the following cohomology rings with coefficients in $\mathbb{F}_{2}$ :

$$
\begin{aligned}
& H^{*}\left(B D_{4}\right) \cong \mathbb{F}_{2}[X, Y] \\
& H^{*}\left(B D_{2^{n}}\right) \cong \mathbb{F}_{2}[X, Y, W] /\left(X^{2}+X Y\right) \text { for } n \geq 3, \\
& H^{*}\left(B Q_{8}\right) \cong \mathbb{F}_{2}[X, Y, V] /\left(X^{2}+X Y+Y^{2}, X^{2} Y+X Y^{2}\right), \\
& H^{*}\left(B Q_{2^{n}}\right) \cong \mathbb{F}_{2}[X, Y, V] /\left(X^{2}+X Y, Y^{3}\right) \text { for } n \geq 4 \text { and } \\
& H^{*}\left(B S D_{2^{n}}\right) \cong \mathbb{F}_{2}[X, Y, U, V] /\left(X^{2}+X Y, X U, X^{3}, U^{2}+\left(X^{2}+Y^{2}\right) V\right) \\
& \quad \text { for } n \geq 4,
\end{aligned}
$$

where $\operatorname{deg}(X)=\operatorname{deg}(Y)=1, \operatorname{deg}(W)=2, \operatorname{deg}(U)=3$ and $\operatorname{deg}(V)=4$ in all the cases.

The results in this paper will not require that the morphisms between the cohomologies of the spaces preserve the structure of the algebra over the Steenrod algebra, but in some proofs we will use that such a structure exists. More precisely we will use that it is unstable and that it is known that $\operatorname{Sq}^{1}(W)=W Y$ in $H^{*}\left(B D_{2^{n}}\right)(n \geq 3)$.

The following properties can be found in BL97. There is a tower of principal fibrations:

$$
\cdots \stackrel{\pi_{n}}{\rightarrow} B D_{2^{n}} \stackrel{\pi_{n}-1}{\rightarrow} B D_{2^{n-1}} \stackrel{\pi_{n}-2}{\rightarrow} \cdots \stackrel{\pi_{3}}{\rightarrow} B D_{8} \stackrel{\pi_{2}}{\rightarrow} B D_{4},
$$

where $\pi_{2}$ is classified by the class $X^{2}+X Y \in H^{2}\left(B D_{4}\right)$ and $\pi_{n}$ by $W \in H^{2}\left(B D_{2^{n}}\right)$ for $n \geq 3$.

The quaternion and semidihedral groups fit in the following central extensions:

$$
\mathbb{Z} / 2 \rightarrow Q_{2^{n}} \rightarrow D_{2^{n-1}} \text { and } \mathbb{Z} / 2 \rightarrow S D_{2^{n}} \rightarrow D_{2^{n-1}},
$$

classified by the following classes in $H^{2}\left(B D_{2^{n-1}}\right): X^{2}+X Y+Y^{2}$ in the case $Q_{8}$, $W+Y^{2}$ in the case $Q_{2^{n}}(n \geq 4)$ and $W+X^{2}$ in the case $S D_{2^{n}}(n \geq 4)$.

\section{Iterated Massey PRoducts in the COHOMOlOGy of MAXimal NILPOTENCY CLASS 2-GROUPS}

This section is devoted to the computation of the iterated Massey products in the cohomology of dihedral, quaternion and semidihedral groups. The results presented here as Lemmas 4.2, 4.3, 4.4 and 4.5 can be summarized in the following theorem:

Theorem 4.1. Consider $D_{2^{n}}(n \geq 2), Q_{2^{n}}(n \geq 3)$ and $S D_{2^{n}}(n \geq 4)$ to be the dihedral, quaternion and semidihedral groups of order $2^{n}$, and consider the generators of their cohomology as denoted in equation (3). Then:

(a) Neither of the elements $W, W+Y^{2}$ or $W+X^{2}$ is contained in an iterated Massey product of degree one elements in the cohomology of $D_{2^{n}}$ of order less than $2^{n-1}$.

(b) The $2^{n-1}$-fold iterated Massey product in the cohomology of $D_{2^{n}}$ defined as $\langle X, X+Y, \ldots, X, X+Y\rangle$ contains $W, W+Y^{2}$ and $W+X^{2}$. 
(c) The $2^{n-1}$ th-fold iterated Massey product in the cohomologies of $D_{2^{n}}, Q_{2^{n}}$ and $S D_{2^{n}}$ defined as $\langle X, X+Y, \ldots, X, X+Y\rangle$ does not contain the zero element.

(d) The $m$-fold iterated Massey product $\langle X, X+Y, X, X+Y, \ldots\rangle$ is not defined for $m>2^{n-1}$ in any of the cohomologies of $D_{2^{n}}, Q_{2^{n}}$ and $S D_{2^{n}}$.

(e) $\left\langle Y, Y^{2}, Y, Y^{2}\right\rangle=\{V\}$ in the cohomology of $Q_{2^{n}}$.

(f) $\left\langle X, X^{2}, Y\right\rangle=\left\{U, U+Y^{3}\right\}$ and $\left\langle X, X^{2}, X, X^{2}\right\rangle=\{V, V+Y U\}$ in the cohomology of $S D_{2^{n}}$.

The proof of this theorem will be done in the next two subsections: the first one is devoted to the computations of iterated Massey products of degree one elements while the second will use the Yoneda cocomplex to prove the last two statements in the theorem.

4.1. Iterated Massey products of degree one elements. The representations described in Appendix $\mathrm{A}$ allow us to compute some iterated Massey products in the cohomology of the dihedral, quaternion and semidihedral groups:

Lemma 4.2. Consider the cohomology of $D_{2^{n}}, n \geq 3$ as denoted in equation (3). Then:

(a) Neither of the elements $W, W+Y^{2}$ or $W+X^{2}$ is contained in an iterated Massey product of degree one elements of order less than $2^{n-1}$.

(b) The $2^{n-1}$-fold iterated Massey product $\langle X, X+Y, \ldots, X, X+Y\rangle$ contains $W$, $W+Y^{2}$ and $W+X^{2}$.

Proof. Assume first that an $m$-fold iterated Massey product contains either $W$, $W+Y^{2}$ or $W+X^{2}$. Then we would have the following diagram:

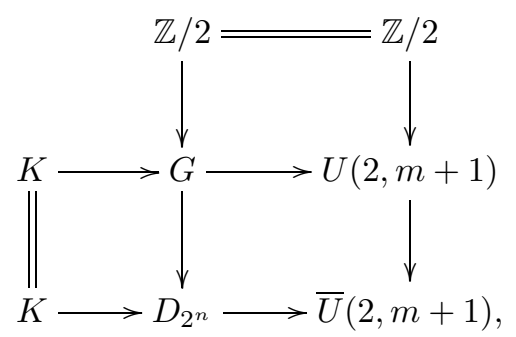

where the bottom right square is a pull-back, $K$ is defined as the kernel of the horizontal arrows and the vertical lines are central extensions.

As either $W, W+Y^{2}$ or $W+X^{2}$ classifies the extension, then $G$ is isomorphic to either $D_{2^{n+1}}, Q_{2^{n+1}}$ or $S D_{2^{n+1}}$. The center of $G$ is exactly $\mathbb{Z} / 2$. If $K$ is nontrivial, as it is a normal subgroup in $G$, then $K$ intersects nontrivially the center of $G$, so it contains the center of $G$. But this implies that the center of $G$ maps injectively to $D_{2^{n}}$, and this contradicts the exactness of the vertical line. This implies that $K$ is trivial, so, there is an injection of $G$ in $U(2, m+1)$, so by Lemma A.4. $m \geq 2^{n-1}$.

The representations in Lemma A.3 and Theorem 2.7 tell us that the $2^{n-1}$-fold iterated Massey product $\langle X, X+Y, \ldots, X, X+Y\rangle$ contains $W, W+Y^{2}$ and $W+$ $X^{2}$.

The following lemma will use the same notation for the generators of $H^{1}(G)$ for different $G$, as noted in equation (3), and the result applies to all of them. 
Lemma 4.3. Consider the cohomology of the dihedral, quaternion and semidihedral groups of order $2^{n}$ as denoted in equation (3). Then, for all these groups:

(a) The $2^{n-1}$-fold iterated Massey product defined as $\langle X, X+Y, \ldots, X, X+Y\rangle$ does not contain the zero element.

(b) The $m$-fold iterated Massey product $\langle X, X+Y, X, X+Y, \ldots\rangle$ is not defined for $m>2^{n-1}$.

Proof. To prove (a), assume that the zero element is in a $2^{n-1}$-fold iterated Massey product of type $\langle X, X+Y, \ldots, X, X+Y\rangle$ of the cohomology of $G$, where $G$ is either $D_{2^{n}}, Q_{2^{n}}$ or $S D_{2^{n}}$. Then there would be a group morphism from $G$ to $\bar{U}\left(\mathbb{F}_{2}, 2^{n-1}+1\right)$ which lifts to a group morphism from $G$ to $U\left(\mathbb{F}_{2}, 2^{n-1}+1\right)$ such that the image of $x$ is a matrix with all the entries in position $(i, i+1)$ equal to 1 . The order of such an element is $2^{n}$, bigger than the order of $x$, getting a contradiction.

(b) can be deduced from (a): if an $m$-fold iterated Massey product $\langle X, X+$ $Y, X, X+Y, \ldots\rangle$ with $m>2^{n-1}$ is defined, then the zero element must be in all the strictly shorter subproducts, in particular in the $2^{n-1}$-fold product of this type.

4.2. Iterated Massey products in higher degrees. Till now all the computations of iterated Massey products have involved only elements in cohomology in degree 1, obtaining elements in degree 2. In this subsection we compute Massey products also involving elements in degree 2 , and obtaining the generators in degrees 3 and 4 which appear in the cohomology of the quaternion and semidihedral groups.

We start with the quaternion groups.

Lemma 4.4. Consider the notation in equation (3) for the cohomology of $Q_{2^{n}}$, the quaternion group of order $2^{n}$ with $n \geq 3$. Then

$$
\left\langle Y, Y^{2}, Y, Y^{2}\right\rangle=\{V\}
$$

Proof. In this proof we will consider $\left(P_{\bullet}, \partial_{\bullet}\right)$, the projective resolution of $\mathbb{F}_{2}$ as an $\mathbb{F}_{2}\left[Q_{2^{n}}\right]$-module described in Lemma B.2. We also consider the generators $Y$ and $V$ in $H^{*}\left(B Q_{2^{n}}\right)$ as elements in the Yoneda cocomplex as computed in Lemma B.3.

With all this data, computing products in these generators can be carried out by composing the corresponding cochain maps, so we can easily find cochain maps representing $Y^{2}$ and $Y^{3}$ :

(i) $Y^{2}$ can be represented by cochain maps $\left(Y^{2}\right)_{i} \stackrel{\text { def }}{=} Y_{i+1} \circ Y_{i}: P_{i+2} \rightarrow P_{i}$, obtaining:

$$
\left(Y^{2}\right)_{4 i}=(10),\left(Y^{2}\right)_{4 i+1}=\left(\begin{array}{l}
1 \\
0
\end{array}\right),\left(Y^{2}\right)_{4 i+2}=\left(\begin{array}{c}
0 \\
N_{x}
\end{array}\right) \text { and }\left(Y^{2}\right)_{4 i+3}=\left(\begin{array}{ll}
0 & N_{x}
\end{array}\right) .
$$

(ii) To compute the cochain maps representing $Y^{3}$ we can use the previous ones in the composition $\left(Y^{3}\right)_{i} \stackrel{\text { def }}{=}\left(Y^{2}\right)_{i+1} \circ Y_{i}: P_{i+3} \rightarrow P_{i}$, obtaining:

$$
\left(Y^{3}\right)_{4 i}=(0),\left(Y^{3}\right)_{4 i+1}=\left(\begin{array}{c}
N_{x} \\
0
\end{array}\right),\left(Y^{3}\right)_{4 i+2}=\left(\begin{array}{cc}
0 & 0 \\
0 & N_{x}
\end{array}\right) \text { and }\left(Y^{3}\right)_{4 i+3}=\left(N_{x} 0\right) .
$$


Now we proceed by computing one element in the 4-fold iterated Massey product $\left\langle Y, Y^{2}, Y, Y^{2}\right\rangle$. We must find the following coefficients in a defining system:

$$
\left(\begin{array}{ccccc}
1 & Y & \alpha & \beta & \\
0 & 1 & Y^{2} & \alpha & \gamma \\
0 & 0 & 1 & Y & \alpha \\
0 & 0 & 0 & 1 & Y^{2} \\
0 & 0 & 0 & 0 & 1
\end{array}\right)
$$

such that $\delta \alpha=Y^{3}, \delta \beta=Y \alpha+\alpha Y, \delta \gamma=Y^{2} \alpha+\alpha Y^{2}$. Note that we just need to compute the maps in low degrees, just enough to compose them and get the product. Now we proceed in the computation of representatives for $\alpha, \beta$ and $\gamma$ :

(i) To compute $\alpha$ we start with $\alpha_{0}=\left(\begin{array}{ll}1 & 0\end{array}\right): P^{2} \rightarrow P^{0}$. Now use the relation $\left(Y^{3}\right)_{0}=(\delta \alpha)_{0}=\alpha_{0} \circ \partial_{3}-\partial_{1} \circ \alpha_{1}$, so $\alpha_{1}$ is a lifting of the $\mathbb{F}_{2}\left[Q_{2^{n}}\right]$-morphism $\left(Y^{3}\right)_{0}+\alpha_{0} \circ \partial_{3}$ (we can avoid the signs as we are working in $\mathbb{F}_{2}$ ). This can be done step by step obtaining $\alpha_{i}: P_{i+2} \rightarrow P_{i}$ :

$$
\alpha_{0}=\left(\begin{array}{ll}
10 & 0
\end{array}\right), \alpha_{1}=\left(\begin{array}{l}
1 \\
0
\end{array}\right), \alpha_{2}=\left(\begin{array}{l}
J \\
L
\end{array}\right) \text { and } \alpha_{3}=\left(\begin{array}{ll}
K & L+N_{x}
\end{array}\right) .
$$

(ii) Computation of $\beta$ is done from the formula $\delta \beta=Y \alpha+\alpha Y$, so we first need $\mathbb{F}_{2}\left[Q_{2^{n}}\right]$-morphisms $(Y \alpha+\alpha Y)_{i}: P_{i+3} \rightarrow P_{i}$ calculated as composition and sum of the previously computed $Y_{j}$ and $\alpha_{k}$, obtaining:

$$
\begin{aligned}
& (Y \alpha+\alpha Y)_{0}=(0),(Y \alpha+\alpha Y)_{1}=\left(\begin{array}{c}
L+N_{x} \\
J
\end{array}\right),(Y \alpha+\alpha Y)_{2}=\left(\begin{array}{cc}
0 & J \\
K & N_{x}
\end{array}\right), \\
& (Y \alpha+\alpha Y)_{3}=\left(L+N_{x} \quad K\right) \text { and }(Y \alpha+\alpha Y)_{4}=(0) \text {. }
\end{aligned}
$$

So we can now proceed to compute a representative for $\beta$ also lifting step by step the corresponding morphisms, using the same technique as that used in the computation in $\alpha . \beta_{i}: P_{i+2} \rightarrow P_{i}$ can be taken as:

$$
\beta_{0}=\left(\begin{array}{ll}
00 \\
0
\end{array}\right), \beta_{1}=\left(\begin{array}{l}
0 \\
0
\end{array}\right), \beta_{2}=\left(\begin{array}{c}
1+J+J^{2} \\
L(1+J)
\end{array}\right) \text { and } \beta_{3}=\left(K L+N_{x}\right) .
$$

(iii) Relation $\delta \gamma=Y^{2} \alpha+\alpha Y^{2}$ gives us the coefficients in the computation of $\gamma$, following the same procedure as the computation of $\beta$. For the sake of completeness we list here $\left(Y^{2} \alpha+\alpha Y^{2}\right)_{i}: P_{i+4} \rightarrow P_{i}$ for small $i$ :

$$
\begin{gathered}
\left(Y^{2} \alpha+\alpha Y^{2}\right)_{0}=(J),\left(Y^{2} \alpha+\alpha Y^{2}\right)_{1}=\left(\begin{array}{cc}
K & L \\
0 & 0
\end{array}\right),\left(Y^{2} \alpha+\alpha Y^{2}\right)_{2}=\left(\begin{array}{ll}
J & 0 \\
L & 0
\end{array}\right) \\
\text { and }\left(Y^{2} \alpha+\alpha Y^{2}\right)_{4}=(K) .
\end{gathered}
$$

Also some possible choices for $\gamma, \gamma_{i}: P_{i+3} \rightarrow P_{i}$, can be taken as:

$$
\gamma_{0}=(0), \gamma_{1}=\left(\begin{array}{l}
0 \\
1
\end{array}\right), \gamma_{2}=\left(\begin{array}{ll}
0 & 1 \\
1 & 0
\end{array}\right) \text { and } \gamma_{3}=\left(\begin{array}{ll}
0 & 1
\end{array}\right) \text {. }
$$

Now we can get an element of the 4-fold iterated Massey product by composing and adding the previous computations in the formula $Y \gamma+\alpha^{2}+\beta Y$. We obtain the $\mathbb{F}_{2}\left[Q_{2^{n}}\right]$-morphism $\left(Y \gamma+\alpha^{2}+\beta Y\right)_{0}: P_{4} \rightarrow P_{0}$ represented by the matrix (1), so it is equivalent to $V$.

This procedure proves that $V \in\left\langle Y, Y^{2}, Y, Y^{2}\right\rangle$, but we have done several choices. Let us now see that if we choose other elements we again get $V$ : by Kra66, Theorem 3 ] we can fix the representatives of $Y$ and $Y^{2}$ to construct any defining system. Assume we change all coefficients $\alpha$ by (possibly) $\alpha^{\prime}, \alpha^{\prime \prime}, \alpha^{\prime \prime \prime}, \beta$ by $\beta^{\prime}$, and finally 
$\gamma$ by $\gamma^{\prime}$ getting a new defining system:

$$
\left(\begin{array}{ccccc}
1 & Y & \alpha^{\prime} & \beta^{\prime} & \\
0 & 1 & Y^{2} & \alpha^{\prime \prime} & \gamma^{\prime} \\
0 & 0 & 1 & Y & \alpha^{\prime \prime \prime} \\
0 & 0 & 0 & 1 & Y^{2} \\
0 & 0 & 0 & 0 & 1
\end{array}\right)
$$

We have that $\delta\left(\alpha-\alpha^{\prime}\right)=0$, so there are $a^{\prime}$ and $b^{\prime}$ in $\mathbb{F}_{2}$ such that

$$
\alpha^{\prime}=\alpha+a^{\prime} X^{2}+b^{\prime} Y^{2} .
$$

The same argument applied to $\alpha^{\prime \prime}$ and $\alpha^{\prime \prime \prime}$ gives us the relations

$$
\alpha^{\prime \prime}=\alpha+a^{\prime \prime} X^{2}+b^{\prime \prime} Y^{2} \text { and } \alpha^{\prime \prime \prime}=\alpha+a^{\prime \prime \prime} X^{2}+b^{\prime \prime \prime} Y^{2},
$$

for $a^{\prime \prime}, a^{\prime \prime \prime}, b^{\prime \prime}$ and $b^{\prime \prime \prime}$ elements in $\mathbb{F}_{2}$.

Now $\delta\left(\beta-\beta^{\prime}\right)=\left(Y \alpha^{\prime \prime}+\alpha^{\prime} Y\right)-(Y \alpha+\alpha Y)=Y\left(\alpha^{\prime \prime}-\alpha\right)+\left(\alpha^{\prime}-\alpha\right) Y=Y\left(a^{\prime \prime} X^{2}+\right.$ $\left.b^{\prime \prime} Y^{2}\right)+\left(a^{\prime} X^{2}+b^{\prime} Y^{2}\right) Y=\left(a^{\prime \prime}+a^{\prime}\right) Y X^{2}+\left(b^{\prime \prime}+b^{\prime}\right) Y^{3}$. As $Y X^{2} \neq 0 \in H^{*}\left(B Q_{2^{n}}\right)$ we get that $a^{\prime \prime}=a^{\prime}$ and there are possibly $a^{(i v)}, b^{(i v)}$ and $c^{(i v)}$ in $\mathbb{F}_{2}$ such that

$$
\beta^{\prime}=\beta+a^{(i v)} X^{2}+b^{(i v)} Y^{2}+c^{(i v)} \alpha .
$$

A similar expansion of the expression $\delta\left(\gamma-\gamma^{\prime}\right)=\left(Y \alpha^{\prime \prime \prime}+\alpha^{\prime \prime} Y\right)-(Y \alpha+\alpha Y)$ tells us that

$$
\gamma^{\prime}=\gamma+a^{(v)} X^{3}+b^{(v)} Y^{3}
$$

So, the result of this defining system differs from $V$ by an element which can be written as, after simplifying, $\alpha\left(a^{\prime \prime \prime} X^{2}+\left(b^{\prime \prime \prime}+1\right) Y^{2}\right)+\left(a^{\prime} X^{2}+b^{\prime} Y^{2}\right) \alpha$, for $a^{\prime}, a^{\prime \prime \prime}, b^{\prime}, b^{\prime \prime \prime} \in \mathbb{F}_{2}$. We again use the Yoneda cocomplex for all these generators to compute it, and this always gives the zero element in cohomology, so

$$
\left\langle Y, Y^{2}, Y, Y^{2}\right\rangle=\{V\} .
$$

Lemma 4.5. Let $S D_{2^{n}}$ be a semidihedral group of order $2^{n}$, with $n \geq 4$. Fix $X$, $Y, U$ and $V$ to be the generators in $H^{*}\left(S D_{2^{n}}\right)$ as in equation (3). Then

$$
\left\langle X, X^{2}, Y\right\rangle=\left\{U, U+Y^{3}\right\} \text { and }\left\langle X, X^{2}, X, X^{2}\right\rangle=\{V, V+Y U\} .
$$

Proof. Here we use the projective resolution $\left(P_{\bullet}, \partial_{\bullet}\right)$ from Lemma B.5 and the properties of the representatives of $X, Y, U$ and $V$ in the Yoneda cocomplex listed in Lemma B.6.

The thread of the proof is the same as the one in Lemma 4.4. To make it shorter and clearer we will focus on the entries of the matrices as $\mathbb{F}_{2}\left[Q_{2^{n}}\right]$-morphisms in the Yoneda cocomplex which define the elements we are interested in.

We must start by checking that $U$ or $U+Y^{2}$ are in $\left\langle X, X^{2}, Y\right\rangle$. By Lemma B.6. this reduces to seeing that the first coordinate, as an $\mathbb{F}_{2}\left[Q_{2^{n}}\right]$-morphism $P_{3} \rightarrow P_{0}$ in the Yoneda cocomplex, in an explicit defining system of this iterated Massey product, is nonzero. We need to compute the first degrees of cochain maps $\alpha$ and $\beta$ fitting in the following defining system:

$$
\left(\begin{array}{cccc}
1 & X & \alpha & \\
0 & 1 & X^{2} & \beta \\
0 & 0 & 1 & Y
\end{array}\right)
$$


(i) The $\mathbb{F}_{2}\left[S D_{2^{n}}\right]$-morphism $\alpha$ must fulfill $\delta \alpha=X^{3}$. A direct computation gives us that we can take $\alpha_{i}: P_{i+2} \rightarrow P_{i}$ as:

$$
\alpha_{0}=\left(\begin{array}{lllll}
1 & 0 & 0
\end{array}\right) \text { and } \alpha_{1}=\left(\begin{array}{cccc}
1+I^{2^{n-1}-3} & t\left(L+I^{2^{n-2}-2}\right) & x^{2} I^{2^{n-1}-5} & 0 \\
0 & 1 & 0 & 0
\end{array}\right) \text {. }
$$

(ii) Observe that $\delta$ must fulfill $\delta \beta=X^{2} Y$ and we have the relation $X^{3}=X^{2} Y$, so we can take $\alpha=\beta$.

So the resulting element in $\left\langle X, X^{2}, Y\right\rangle$ of this defining system is $X \alpha+\alpha Y=X \alpha+$ $\beta Y$, and we just need to compute the evaluation of the morphism at level 0 :

$$
\varepsilon\left(\alpha_{0} X_{2}+Y_{0} \alpha_{1}\right)=(1010),
$$

as a map from $P_{3}$ to $\mathbb{F}_{2}$. By Lemma B.6. this element corresponds either to $U+Y^{3}$ or $U$.

Now we must deal with the indeterminacy in this 3-fold iterated Massey product to get the final result. Applying May69, Proposition 2.3], in a 3-fold iterated Massey product, two elements in $\left\langle X, X^{2}, Y\right\rangle$ differ by an element of the form $\langle Z, Y\rangle+\left\langle X, Z^{\prime}\right\rangle$, for $Z, Z^{\prime}$ elements in $H^{2}\left(B S D_{2^{n}}\right)$. As $Y^{3}$ is the only element which can be constructed in this way we get

$$
\left\langle X, X^{2}, Y\right\rangle=\left\{U, U+Y^{3}\right\} .
$$

We now need to compute one element in $\left\langle X, X^{2}, X, X^{2}\right\rangle$. Again, the computations will focus on the information which tells us that either $V$ or $V+Y U$ belongs to this 4-fold iterated Massey product: that is, by Lemma B.6, there is an element of the form $(1 * * * 0)$ in $\left\langle X, X^{2}, X, X^{2}\right\rangle$, seen as a map from $P_{4}$ to $\mathbb{F}_{2}$ in the Yoneda cocomplex.

Here we use the same procedure as in the proof of Lemma 4.4, using the matrices $X_{i}$ described above. We need $\alpha, \beta$ and $\gamma$ in a defining system:

$$
\left(\begin{array}{ccccc}
1 & X & \alpha & \beta & \\
0 & 1 & X^{2} & \alpha & \gamma \\
0 & 0 & 1 & X & \alpha \\
0 & 0 & 0 & 1 & X^{2} \\
0 & 0 & 0 & 0 & 1
\end{array}\right)
$$

So the relations we must care about are $\delta \alpha=X^{3}, \delta \beta=X \alpha+\alpha X$ and $\delta \gamma=$ $X^{2} \alpha+\alpha X^{2}$.

By definition, $\alpha$ may be taken to be the same $\alpha$ considered in the computation of $\left\langle X, X^{2}, Y\right\rangle$. Moreover, as $X \alpha+\alpha X$ gives the same representative as $X^{3}$ in the Yoneda cocomplex, we can also take $\beta=\alpha$. Finally, this election of $\alpha$ makes $X^{2} \alpha+\alpha X^{2}$ to be the zero element, so we can take $\gamma=0$.

Now we can proceed in the computation of the first coordinate of $X \gamma+\alpha^{2}+\beta X^{2}$ :

$$
\varepsilon\left(0+\alpha_{0} \alpha_{2}+X_{0} X_{1} \alpha_{2}\right) .
$$

We just need the first and last columns of $\alpha_{2}$, which we can see have $(1,0,0)$ and $(0,0,0)$ as coefficients respectively. As the result of the computations is the sum of the first two rows, we get that the result is of the form $(1 * * * 0)$ as a map from $P_{4}$ to $\mathbb{F}_{2}$, obtaining either $V$ or $V+Y U$ by Lemma B.3.

We can also use the computations in the indeterminacy of the 4-fold Massey iterated product in Lemma 4.4 to get a formula for the indeterminacy in this 4fold iterated Massey product. Two elements in $\left\langle X, X^{2}, X, X^{2}\right\rangle$ differ by an element 
which can be expressed as $\alpha\left(a X^{2}+b Y^{2}\right)+\left(a^{\prime} X^{2}+b^{\prime} Y^{2}\right) \alpha$, with $\alpha$ a cochain such that $\delta \alpha=X^{3}$ and $a, b, a,{ }^{\prime} b^{\prime} \in \mathbb{F}_{2}$. Any element of this form gives a map $\mathbb{F}_{2}[G]^{5} \rightarrow \mathbb{F}_{2}[G] \rightarrow \mathbb{F}_{2}$ with zeros in the first and last coordinates and the coefficient $a^{\prime}$ in the second. So just the element $Y U$ is in the indeterminacy, getting that:

$$
\left\langle X, X^{2}, X, X^{2}\right\rangle=\{V, V+Y U\} .
$$

\section{Cohomological uniqueness}

This section is divided into three subsections with the same structure, one for each family of 2-groups of maximal nilpotency class.

5.1. Dihedral groups. Consider $D_{2^{n}}$, the dihedral group of order $2^{n}$, and its cohomology for $n \geq 3$ as defined in equations (2) and (3).

The following lemma uses the fact that, for any topological space $\mathcal{X}, H^{*}(\mathcal{X})$ is an unstable algebra over the Steenrod algebra, but the lemma does not require that this structure must be the same as the one in $H^{*}\left(B D_{2^{n}}\right)$ :

Lemma 5.1. Let $\mathcal{X}$ be a space such that $H^{*}(\mathcal{X}) \cong H^{*}\left(B D_{2^{n}}\right)$ as algebras. Let $\phi: \mathcal{X} \rightarrow B D_{2^{n}}$ be a map inducing the identity in degree one cohomology. Then either $\phi^{*}$ is an isomorphism or $\phi^{*}(W)=0$.

Proof. According to the hypothesis, $\phi^{*}$ is an isomorphism if and only if $W$ is in the image of $\phi^{*}$. In other words $\phi^{*}$ is not an isomorphism if and only if $\phi^{*}(W)=$ $a X^{2}+b Y^{2}$ for $a, b \in \mathbb{F}_{2}$. Assume then that $\phi^{*}(W)=a X^{2}+b Y^{2}$. Applying $\mathrm{Sq}^{1}$ in both sides we get $\operatorname{Sq}^{1}(W)=W Y$ and $\operatorname{Sq}^{1}\left(a X^{2}+b Y^{2}\right)=0$. So $0=\operatorname{Sq}^{1}\left(\phi^{*}(W)\right)=$ $\phi^{*}\left(\mathrm{Sq}^{1}(W)\right)=\left(a X^{2}+b Y^{2}\right) Y$, which implies that $a=b=0$.

Theorem 5.2. $B D_{2^{n}}$ is determined by its $\mathcal{M}$-cohomology.

Proof. Fix $\mathcal{X}$ to be a 2-complete topological space having the homotopy type of a $\mathrm{CW}$-complex and $\mathcal{M}$-comparable to $B D_{2^{n}}$.

For $n=2, D_{4} \cong \mathbb{Z} / 2 \times \mathbb{Z} / 2$ and the result follows from Proposition 2.6.

Assume that $n \geq 3$. Then we should give a map $\phi_{n}: \mathcal{X} \rightarrow B D_{2^{n}}$ inducing an isomorphism in cohomology up to degree 2. Consider the tower of principal fibrations in equation (44), where each $\pi_{k}$ corresponds to the central extension:

$$
1 \rightarrow \mathbb{Z} / 2 \rightarrow D_{2^{k+1}} \rightarrow D_{2^{k}} \rightarrow 1
$$

classified either by $X^{2}+X Y$ if $k=2$ or by $W$ when $k>2$.

Consider $\phi_{2}: \mathcal{X} \rightarrow B D_{4}$, a map representing the classes $X$ and $Y$. The composite

$$
\mathcal{X} \stackrel{\phi_{2}}{\longrightarrow} B D_{4} \stackrel{X^{2}+X Y}{\longrightarrow} K(\mathbb{Z} / 2,2)
$$

is nullhomotopic, so $\phi_{2}$ factors as a composition $\pi_{2} \circ \phi_{3}$, with $\phi_{3}: \mathcal{X} \rightarrow B D_{8}$.

Now, if we assume that $\phi_{k}: \mathcal{X} \rightarrow B D_{2^{k}}$ inducing the identity in $H^{1}$ is defined, this map will extend to a map $\phi_{k+1}: \mathcal{X} \rightarrow B D_{2^{k+1}}$ if and only if $\phi_{k}^{*}(W)=0$. Using Lemmas A.4 and 5.1, and the fact that $\left(2^{k}-2\right)$-fold iterated Massey products must fulfill

$$
\phi_{k}^{*}\left(\langle X, X+Y, X, X+Y, \cdots\rangle_{H^{*}\left(B D_{2^{k}}\right)}\right) \subset\langle X, X+Y, X, X+Y, \cdots\rangle_{H^{*}(\mathcal{X})}
$$

we get that $\phi_{k}^{*}(W)=0$ for all $k<n$ (the subscripts in the formula indicate the algebra where the $\left(2^{k}-2\right)$-fold iterated Massey products are considered). So, the map $\phi_{k}$ extends to $\phi_{k+1}: \mathcal{X} \rightarrow B D_{2^{k+1}}$ for $k<n$. 
It remains to check the last step: if $\phi_{n}(W)=0$, then it extends to $\phi_{n+1}$. Such a map $\phi_{n+1}: \mathcal{X} \rightarrow B D_{2^{n+1}}$ inducing the identity in $H^{1}$ cannot exist because the $2^{n+1}$ fold iterated Massey product $\langle X, X+Y, X, X+Y, \cdots\rangle$ is defined in $H^{*}\left(B D_{2^{n+1}}\right)$ and it is not defined in $H^{*}(\mathcal{X})$ by Lemma A.4

So $\phi_{n}^{*}(W) \neq 0$, and by Lemma 5.1, $\phi_{n}$ is an isomorphism in cohomology and $\mathcal{X} \simeq B D_{2^{n}}$.

\subsection{Quaternion groups.}

Lemma 5.3. Consider the notation in equation (3) for the cohomology of the dihedral and quaternion groups. Let $\mathcal{X}$ be a space $\mathcal{M}$-comparable to $B Q_{2^{n}}$, and $\phi: \mathcal{X} \rightarrow B D_{2^{r}}$ be a map such that $\phi^{*}(X)=X$ and $\phi^{*}(Y)=Y$. Then $\phi^{*}(W)=0$ or $\phi^{*}(W)=Y^{2}$.

Proof. Recall that $\left\{X^{2}, Y^{2}\right\}$ is a basis of $H^{2}(\mathcal{X})$. Then there are $a, b \in \mathbb{F}_{2}$ such that $\phi^{*}(W)=a X^{2}+b Y^{2}$. If we apply $\mathrm{Sq}^{1}$ to both sides of the identity we get $a X^{2} Y=0$ (notice $Y^{3}=0$ in $H^{*}(\mathcal{X})$ ), getting the desired result.

Theorem 5.4. $B Q_{2^{n}}$ is determined by its $\mathcal{M}$-cohomology.

Proof. Let $\mathcal{X}$ be a 2-complete space having the homotopy type of a CW-complex and $\mathcal{M}$-comparable to $B Q_{2^{n}}$.

We must consider the cases $n=3$ and $n \neq 3$ separately.

Consider $Q_{8}$ and its cohomology as in equations (2) and (3). Let $\phi_{2}: \mathcal{X} \rightarrow$ $B(\mathbb{Z} / 2 \times \mathbb{Z} / 2)$ be the map representing the elements $X$ and $Y$ in cohomology. Such a map factors through $\phi_{3}: \mathcal{X} \rightarrow Q_{8}$ because $\phi_{2}^{*}\left(X^{2}+X Y+Y^{2}\right)=0$, so we have a map $\phi_{3}$ inducing the identity in $H^{1}$. Now use Lemma 4.4 to get that it must be an isomorphism in cohomology, so a homotopy equivalence.

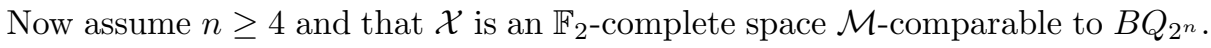
Let $\phi_{2}: \mathcal{X} \rightarrow B(\mathbb{Z} / 2 \times \mathbb{Z} / 2)$ be the map representing the elements $X$ and $Y$ in cohomology. Consider the tower of principal fibrations

$$
\cdots \rightarrow B D_{2^{k}} \stackrel{\pi_{k-1}}{\rightarrow} B D_{2^{k-1}} \stackrel{\pi_{n-2}}{\rightarrow} \cdots \stackrel{\pi_{3}}{\rightarrow} B D_{8} \stackrel{\pi_{2}}{\rightarrow} B(\mathbb{Z} / 2 \times \mathbb{Z} / 2) .
$$

Recall that the map $\pi_{2}$ is classified by the class $X^{2}+X Y$ and each $\pi_{i}$ for $i \geq 3$ is classified by $W$.

Since $\phi_{2}^{*}\left(X^{2}+X Y\right)=0, \phi_{2}$ lifts to $\phi_{3}: \mathcal{X} \rightarrow B D_{8}$, a map such that $\phi_{3}^{*}(X)=X$ and $\phi_{3}^{*}(Y)=Y$.

Now assume that $k \geq 3$ and $\phi_{k}: \mathcal{X} \rightarrow B D_{2^{k}}$ such that $\phi_{k}^{*}(X)=X, \phi_{k}^{*}(Y)=Y$. We show that $\phi_{k}^{*}(W)=0$ when $k<n-1$, which implies that $\phi_{k}$ lifts to $\phi_{n-1}: \mathcal{X} \rightarrow$ $B D_{2^{n-1}}$ such that $\phi_{n-1}^{*}(X)=X, \phi_{n-1}^{*}(Y)=Y$.

If $\phi_{k}^{*}(W) \neq 0$, then $\phi_{k}^{*}(W)=Y^{2}$ by Lemma 5.3. This implies $\phi_{k}^{*}\left(Y^{2}+W\right)=0$ and there is a map $\tilde{\phi}_{k+1}: \mathcal{X} \rightarrow B Q_{2^{k+1}}$ which is an isomorphism in cohomology up to degree 3. This implies, by Lemma 2.3, that both $\mathcal{X}$ and $B Q_{2^{k+1}}$ have the same iterated Massey products involving degree one elements. But by Lemma 4.3 . as $k+1<n$, there are iterated Massey products in $H^{*}(\mathcal{X})$ which are not defined in $H^{*}\left(B Q_{2^{k+1}}\right)$, getting a contradiction to the assumption $\phi_{k}^{*}(W) \neq 0$.

It remains to see that $\phi_{n-1}$ lifts to $\phi_{n}: \mathcal{X} \rightarrow B Q_{2^{n}}$, i.e. that $\phi_{n-1}(W)=Y^{2}$ : if $\phi_{n-1}(W) \neq Y^{2}$, then $\phi_{n-1}(W)=0$ by Lemma 2.3. and we get a map $\tilde{\phi}_{n}: \mathcal{X} \rightarrow$ $D_{2^{n}}$. If such a $\tilde{\phi}_{n}$ exists, using again by Lemma 2.3. there exists either a map $\tilde{\phi}_{n+1}: \mathcal{X} \rightarrow D_{2^{n+1}}$ or a map $\tilde{\phi}_{n+1}^{\prime}: \mathcal{X} \rightarrow Q_{2^{n+1}}$, but neither $\tilde{\phi}_{n+1}$ nor $\tilde{\phi}_{n+1}^{\prime}$ can exist because in the cohomology of both targets there are $2^{n}$-fold iterated Massey 
products of type $\langle X, X+Y, X, \ldots, X+Y\rangle$ which are not defined in $H^{*}(\mathcal{X})$ by Lemma 4.3, getting a contradiction.

This implies that we have a map $\phi_{n}: \mathcal{X} \rightarrow B Q_{2^{n}}$ which is the identity in cohomology till degree 3, and by Lemma 4.4, $\phi_{n}^{*}(V)=V$. Then $\phi_{n}^{*}$ is an isomorphism and $\phi_{n}$ is a homotopy equivalence.

Remark 5.5. Observe that iterated Massey products involving elements in degree greater than one cannot be avoided. Consider, as in [Ben92], $\mathcal{X}=S^{3} / Q_{2^{n}} \times B S^{3}$, where the action of $Q_{2^{n}}$ is by left multiplication on $S^{3}$ and where one considers $Q_{2^{n}}$ as a discrete subgroup of the Lie group $S^{3}$.

The space $\mathcal{X}$ is 2-good, and using [Ben92, we have that $H^{*}(\mathcal{X}) \cong H^{*}\left(B Q_{2^{n}}\right)$ as $\mathbb{F}_{2}$-algebras. Moreover, the iterated products of degree one elements are the same: all the information about the iterated Massey products of elements in degree one can be read in the first three steps of a minimal projective resolution of $\mathbb{F}_{2}\left[Q_{2^{n}}\right]$, and the three steps agree with the minimal projective resolution of $C^{*}\left(\mathcal{X}, \mathbb{F}_{2}\right)$. Finally, these are not homotopy equivalent spaces up to 2-completion because, for example, they have different homotopy groups.

5.3. Semidihedral groups. Consider $S D_{2^{n}}$, a semidihedral group of order $2^{n}$, and its cohomology with the notation in equations (22) and (3).

Lemma 5.6. Let $\mathcal{X}$ be a topological space $\mathcal{M}$-comparable to $B S D_{2^{n}}$, and $\phi: \mathcal{X} \rightarrow$ $B D_{2^{r}}(r \geq 3)$ be a map such that $\phi^{*}(X)=X$ and $\phi^{*}(Y)=Y$. Then $\phi^{*}(W)=0$ or $\phi^{*}(W)=X^{2}$.

Proof. Consider $\left\{X^{2}, Y^{2}\right\}$ as a basis of $H^{2}(\mathcal{X})$ as an $\mathbb{F}_{2}$ vector space. Then there are $a$ and $b$ in $\mathbb{F}_{2}$ such that $\phi^{*}(W)=a X^{2}+b Y^{2}$. If we apply $\mathrm{Sq}^{1}$ to both sides of the identity we get $b Y^{3}=0$, getting the desired result.

Theorem 5.7. $B S D_{2^{n}}$ is determined by its $\mathcal{M}$-cohomology.

Proof. Fix $\mathcal{X}$ to be a 2-complete space having the homotopy type of a $C W$-complex and $\mathcal{M}$-comparable to $B S D_{2^{n}}(n \geq 4)$.

Consider $\phi_{2}: \mathcal{X} \rightarrow B D_{4}$ to be a map representing $X$ and $Y$. As $\phi_{2}^{*}\left(X^{2}+X Y\right)=$ 0 , this map will factor through a map $\phi_{3}: \mathcal{X} \rightarrow B D_{8}$ such that $\phi_{3}^{*}: H^{1}\left(B D_{8}\right) \rightarrow$ $H^{1}(\mathcal{X})$ is the identity.

Now assume that we have a map $\phi_{k}: \mathcal{X} \rightarrow B D_{2^{k}}$ which induces the identity in $H^{1}$ and with $k<n-1$. Then, by Lemma 5.6. $\phi_{k}^{*}(W)=X^{2}$ or $\phi_{k}^{*}(W)=0$.

In the first case, $\phi_{k}^{*}\left(W+X^{2}\right)=0$, so there is a map $\tilde{\phi}_{k+1}: \mathcal{X} \rightarrow B S D_{2^{k+1}}$ which is the identity in cohomology in degrees one and two. So, by Lemma 2.3. they must have the same iterated Massey products of degree one elements. But, by Lemma 4.3, if $k+1<n$ there are $2^{n-1}$-fold iterated Massey products which are defined in the cohomology of $\mathcal{X}$, but not in $H^{*}\left(B S D_{2^{b+1}}\right)$, and this contradicts Lemma 2.3.

So we are in the second case and we have a map $\phi_{k+1}: \mathcal{X} \rightarrow B D_{2^{k+1}}$ inducing the identity in $H^{1}$. This procedure can be done up to $\phi_{n-1}: \mathcal{X} \rightarrow B D_{2^{n-1}}$ inducing the identity in $H^{1}$. Again, by Lemma 5.6, $\phi_{n-1}^{*}(W)=0$ or $\phi_{n-1}^{*}(W)=X^{2}$.

In the first case, we obtain a map $\tilde{\phi}_{n}: \mathcal{X} \rightarrow B D_{2^{n}}$ inducing the identity in $H^{1}$. By the previous arguments such a map induces a map either $\tilde{\phi}_{n+1}: \mathcal{X} \rightarrow B D_{2^{n+1}}$ or $\tilde{\phi}_{n+1}: \mathcal{X} \rightarrow B S D_{2^{n+1}}$ inducing the identity in $H^{1}$, and this cannot happen because Lemmas 4.2 and 4.3 imply that there are iterated Massey products of degree one elements defined in $H^{*}\left(B D_{2^{n+1}}\right)$ and $H^{*}\left(B S D_{2^{n+1}}\right)$ which are not defined in $H^{*}(\mathcal{X})$. 
So $\phi_{n-1}^{*}(W)=X^{2}$ and we have a map $\phi_{n}: \mathcal{X} \rightarrow B S D_{2^{n}}$ inducing the identity in $H^{1}$. Now we use Lemma 4.5 to see that it must be an isomorphism in cohomology, and therefore a homotopy equivalence: as $\phi_{n}^{*}\left(\left\{U, U+Y^{3}\right\}\right)=\phi_{n}^{*}\left(\left\langle X, X^{2}, Y\right\rangle\right) \subset$ $\left\langle X, X^{2}, Y\right\rangle=\left\{U, U+Y^{3}\right\}$ we get that either $\phi_{n}^{*}(U)=U$ or $\phi_{n}^{*}\left(U+Y^{3}\right)=U$, so $U$ is in the image of $\phi_{n}^{*}$. Using the same argument applied to $\left\langle X, X^{2}, X, X^{2}\right\rangle=$ $\{V, V+Y U\}$ we get that $V$ is in the image of $\phi_{n}^{*}$. This implies that all generators are in the image, and, up to degree 4 all are finite-dimensional vector spaces, so an epimorphism is an isomorphism.

\section{Appendix A. Representations of maximal nilpotency Class 2-GRoups}

In this section we obtain an explicit minimal degree faithful representation of maximal nilpotency class 2-groups on $\bar{U}\left(\mathbb{F}_{2}, n\right)$ which will allow us to compute iterated Massey products of some degree one elements. The images of the generators of each group are defined using the matrices described below:

Consider $A_{n}$ to be the $2^{n} \times 2^{n}$ matrix defined inductively:

$$
A_{0}=(1), \quad A_{n}=\left(\begin{array}{c|c}
A_{n-1} & A_{n-1} \\
\hline 0 & A_{n-1}
\end{array}\right),
$$

where 0 means a matrix with all the entries equal to zero.

Consider also the $2^{n} \times 2^{n}$-matrix $B_{n}$ with entries $b_{i, j}$ :

$$
b_{i, j} \stackrel{\text { def }}{=} \begin{cases}1 & \text { if } i=j \text { or } j=i+1, \\ 0 & \text { otherwise. }\end{cases}
$$

Finally consider the $2^{n} \times 2^{n}$-matrix $C_{n}$ with entries $c_{i, j}$ :

$$
c_{i, j} \stackrel{\text { def }}{=} \begin{cases}1 & \text { if } i=j, \\ 1 & \text { if } i=1 \text { and } j=2^{n}, \\ 0 & \text { otherwise. }\end{cases}
$$

Most of the proofs will be done by induction, so we need to express matrices $B_{n}$ and $C_{n}$ as a construction of $2^{n-1} \times 2^{n-1}$ matrices: define the $2^{n} \times 2^{n}$ matrix $\Delta_{n}$ as the matrix with all entries 0 except the entry in position $\left(1,2^{n}\right)$ which is 1 . Then:

$$
B_{n}=\left(\begin{array}{c|c}
B_{n-1} & \Delta_{n-1}^{T} \\
\hline 0 & B_{n-1}
\end{array}\right) \text { and } C_{n}=\left(\begin{array}{c|c}
\mathrm{Id} & \Delta_{n-1} \\
\hline 0 & \mathrm{Id}
\end{array}\right),
$$

where $\Delta_{n-1}^{T}$ means the transpose of $\Delta_{n-1}$ and Id the identity matrix in the corresponding rank.

The following calculation gives the property needed to compute the iterated Massey products:

Lemma A.1. Let $B$ be a matrix in $\mathrm{GL}_{m}\left(\mathbb{F}_{2}\right)$ as defined in equation (7) (now $m$ is not necessarily of the form $\left.2^{n}\right)$. Then:

(a) The order of $B$ is $2^{n}$, where $2^{n-1}<m \leq 2^{n}$.

(b) If $l$ is a positive integer such that $2^{l}<\operatorname{Order}(B)$, then the coefficients in the diagonal and in positions $\left(i, i+2^{l}\right)$ in $B^{2^{l}}$ are equal to 1 (for $i$ from 1 to $m-2^{l}$ ) and 0 in all other positions.

Proof. $B$ is the sum of the identity Id and a nilpotent matrix $N$. Coefficients of $N^{a}$ are equal to 0 in positions $(i, k)$ for $k \neq i+a$, and equal to 1 in positions $(i, i+a)$. In particular $N^{a}$ is not 0 for $a<m$ and is 0 for $a \geq m$. Now, as Id and $N$ commute, we can compute $(\operatorname{Id}+N)^{l}$ using the mod 2 binomial formula. In 
particular, $B^{2^{l}}=(\mathrm{Id}+N)^{2^{l}}=\mathrm{Id}+N^{2^{l}}$. Using the description of $N^{a}$ described just before, we get (b). Moreover this tells us that $B^{2^{n}}=\mathrm{Id}$ and that $B^{2^{n-1}} \neq \mathrm{Id}$, where $n$ is the integer considered in (a), and we get the desired result.

Lemma A.2. Fix $n \geq 1$. The matrices $A_{n}, B_{n}$ and $C_{n}$ defined in equations (6), (17) and (8) have the following properties:

(a) $A_{n}^{2}=\mathrm{Id}$,

(b) $A_{n} \Delta_{n}^{T} A_{n}$ is a matrix with all entries equal to 1 ,

(c) $A_{n} B_{n} A_{n}$ has all the entries over the diagonal equal to 1 ,

(d) $\left(A_{n} B_{n}\right)^{2}=$ Id,

(e) the first row of $A_{n} B_{n}$ is equal to the vector $(10 \cdots 0)$, and

(f) $C_{n}$ is in the center of the invertible upper triangular matrices, so, in particular, commutes with $A_{n}$ and $B_{n}$.

Proof. (a) This can be seen by induction on $n$ and the fact that all coefficients are taken in $\mathbb{F}_{2}$ : the case $n=0$ follows directly, and we assume the result is true up to $n-1$. Then:

$$
A_{n}^{2}=\left(\begin{array}{c|c}
A_{n-1} & A_{n-1} \\
\hline 0 & A_{n-1}
\end{array}\right)^{2}=\left(\begin{array}{c|c}
A_{n-1}^{2} & 2 A_{n-1} \\
\hline 0 & A_{n-1}^{2}
\end{array}\right)=\mathrm{Id},
$$

where we are using that, as we are working in $\mathbb{F}_{2}, 2 A_{n-1}=0$.

(b) This can be proved by induction, proceeding as in (a) and using that

$$
\Delta_{n}^{T}=\left(\begin{array}{c|c}
0 & 0 \\
\hline \Delta_{n-1}^{T} & 0
\end{array}\right)
$$

where, in this case, 0 is the $2^{n-1} \times 2^{n-1}$ matrix with all entries equal to 0 .

(c) Here we can also prove it by induction: the case $n=0$ is direct. Assume the result is true up to $n-1$. Then:

$$
A_{n} B_{n} A_{n}=\left(\begin{array}{c|c}
A_{n-1} B_{n-1} A_{n-1} & A_{n-1} \Delta_{n-1}^{T} A_{n-1} \\
\hline 0 & A_{n-1} B_{n-1} A_{n-1}
\end{array}\right) .
$$

We can now use the induction hypothesis and (b) to get the result.

(d) We can write $\left(A_{n} B_{n}\right)^{2}=\left(A_{n} B_{n} A_{n}\right) B_{n}$ and use (c): the position $(i, j)$ of the result of this multiplication is the sum of all entries in the column $j$ of $B_{n}$ from row $i$ on, so this is 1 in the diagonal, and 0 in all other positions, either because we are adding 0 or two $1 \mathrm{~s}$, which is 0 in $\mathbb{F}_{2}$.

(e) Observe that the first row of $A_{n}$ has all coefficients equal to 1 , so the first row of $A_{n} B_{n}$ is, in each position, the sum of all the entries in the corresponding column, so 1 is in the first position and 0 is in the other.

(f) If $U$ is an invertible upper triangular $2^{n} \times 2^{n}$ matrix, the matrix $U C_{n}$ has the same entries as $U$ except for the last element of the first row, which changes by +1 . Multiplying by $C_{n} U$, then we have to add 1 to the first element of the last column, getting the same result. 
Now we will use the matrices $A_{n}, B_{n}$ and $C_{n}$ to construct the following $\left(2^{n}+\right.$ 1) $\times\left(2^{n}+1\right)$ matrices:

$$
\begin{aligned}
& x_{n} \stackrel{\text { def }}{=}\left(\begin{array}{c|cccc}
1 & 1 & 0 & \cdots & 0 \\
\hline 0 & & & & \\
\vdots & & & B_{n} & \\
0 & & & &
\end{array}\right), y_{n} \stackrel{\text { def }}{=}\left(\begin{array}{cc|ccc}
1 & 0 & \cdots & 0 \\
\hline 0 & & & \\
\vdots & & & A_{n} & \\
0 & & &
\end{array}\right), \\
& z_{n} \stackrel{\text { def }}{=}\left(\begin{array}{c|ccc|ccccc}
1 & 0 & \cdots & 0 & 0 & \cdots & 0 & 1 & 0 \\
\hline 0 & & & & & & & \\
\vdots & & A_{n-1} & & & A_{n-1} C_{n-1} & \\
0 & & & & & & & \\
\hline 0 & & & & & & & \\
\vdots & & 0 & & & A_{n-1} &
\end{array}\right) \text { and } \\
& t_{n} \stackrel{\text { def }}{=}\left(\begin{array}{c|ccc|ccc}
1 & 0 & \cdots & 0 & 0 & \cdots & 0 \\
\hline 0 & & & & & \\
\vdots & & A_{n-1} & & A_{n-1} C_{n-1} \\
0 & & & & \\
\hline 0 & & & & \\
\vdots & & 0 & & A_{n-1}
\end{array}\right) .
\end{aligned}
$$

Lemma A.3. The $\left(2^{n}+1\right) \times\left(2^{n}+1\right)$ matrices $x_{n}, y_{n}, z_{n}$ and $t_{n}$ defined above have the following properties:
(a) $x_{n}$ has order $2^{n+1}$,
(b) $y_{n}$ has order 2 ,
(c) $y_{n} x_{n} y_{n}=x_{n}^{-1}$,
(d) $z_{n}^{2}=x_{n}^{2^{n}}$, in particular $z_{n}$ has order 4 ,
(e) $z_{n} x_{n} z_{n}^{-1}=x_{n}^{-1}$
(f) $t_{n}$ has order 2 and
(g) $t_{n} x_{n} t_{n}^{-1}=x_{n}^{2^{n}-1}$.

So $x_{n}$ and $y_{n}$ generate a subgroup isomorphic to $D_{2^{n+2}}, x_{n}$ and $z_{n}$ a subgroup isomorphic to $Q_{2^{n+2}}$, and $x_{n}$ and $t_{n}$ a subgroup isomorphic to $S D_{2^{n+2}}$.

Proof. (a) The order of $x_{n}$ has been computed in Lemma A.1

(b) This is a direct computation using Lemma A.2 (a).

(c) This is equivalent to showing that $\left(y_{n} x_{n}\right)^{2}=\mathrm{Id}$. We have

$$
\left(y_{n} x_{n}\right)^{2}=\left(\begin{array}{c|cccc}
1 & 1 & 0 & \cdots & 0 \\
\hline 0 & & & A_{n} B_{n} &
\end{array}\right)^{2}=\left(\begin{array}{c|c}
1 & 0 \\
\hline 0 & \left(A_{n} B_{n}\right)^{2}
\end{array}\right)=\mathrm{Id},
$$

where we have used Lemma A.2 (e) in the second equality to get zeros in the first row, and Lemma A.2 (c) gives us the last step.

(d) The computation of $x_{n}^{2^{n}}$ has been done in Lemma A.1

$$
x_{n}^{2^{n}}=\left(\begin{array}{c|cccc}
1 & 0 & \cdots & 0 & 1 \\
\hline 0 & \multicolumn{4}{|c}{\text { Id }}
\end{array} .\right.
$$


A direct calculation gives us the expression:

$$
z_{n}^{2}=\left(\begin{array}{c|c|c}
1 & 0 & 0 \quad \cdots \quad 0 \quad 1 \\
\hline 0 & A_{n-1}^{2} & A_{n-1}^{2} C_{n-1}+A_{n-1} C_{n-1} A_{n-1} \\
\hline 0 & 0 & A_{n-1}^{2}
\end{array}\right) .
$$

Now apply Lemma A.2 (a) to obtain the identity in the diagonal and Lemma A.2 (e) to get $A_{n-1}^{2} C_{n-1}+A_{n-1} C_{n-1} A_{n-1}=C_{n-1}+C_{n-1}=0$.

(e) Using (b) and (c) we have to prove that $z_{n} x_{n} z_{n}^{-1}=y_{n}^{-1} x_{n} y_{n}$, which is the same as proving that $\left(y_{n} z_{n}\right) x_{n}=x_{n}\left(y_{n} z_{n}\right)$. Direct computations show us the following results:

$$
y_{n} z_{n}=\left(\begin{array}{c|c|ccccc}
1 & 0 & 0 & \cdots & 0 & 1 & 0 \\
\hline 0 & \text { Id } & \multicolumn{4}{|c}{\Delta_{n-1}} \\
\hline 0 & 0 & \multicolumn{4}{|c}{\text { Id }}
\end{array}\right),
$$

where we have used that $C_{n-1}+\mathrm{Id}=\Delta_{n-1}$.

Observe now that, as $C_{n-1}$ and Id commute with $B_{n-1}$, so does $\Delta_{n-1}$, and use it to check:

$$
\left(y_{n} z_{n}\right) x_{n}=\left(\begin{array}{c|cccc|ccccc}
1 & 1 & 0 & \cdots & 0 & 0 & \cdots & 0 & 1 & 1 \\
\hline 0 & \multicolumn{1}{|c|}{B_{n-1}} & & \Delta_{n-1}^{T}+\Delta_{n-1} B_{n-1} \\
\hline 0 & \multicolumn{1}{|c|}{0} & B_{n-1}
\end{array}\right)=x_{n}\left(y_{n} z_{n}\right) .
$$

(f) The computation of $t_{n}^{2}$ is analogous to the computation of $z_{n}^{2}$ with the first row as the identity matrix, getting $t_{n}^{2}=\mathrm{Id}$.

(g) The statement is equivalent to showing that $\left(t_{n} x_{n}\right)^{2}=x^{2^{n}}$. Directly multiplying the matrices we have:

$$
t_{n} x_{n}=\left(\begin{array}{c|cccc}
1 & 1 & 0 & \cdots & 0 \\
\hline 0 & A_{n-1} B_{n-1} & A_{n-1} \Delta_{n-1}^{T}+A_{n-1} C_{n-1} B_{n-1} \\
\hline 0 & 0 & B_{n-1}
\end{array}\right) .
$$

Now use almost the same computations as in the proof of (c) to show that $\left(t_{n} x_{n}\right)^{2}$ has the same entries as $x_{n}^{2^{n}}$ : we use that the first row of $A_{n-1} \Delta_{n-1}^{T}$ is $(10 \cdots 0)$, and that $A_{n-1} C_{n-1} B_{n-1}=A_{n-1} B_{n-1} C_{n-1}$, so by Lemma A.2 (e) and the multiplication by $C_{n-1}$, the first row of $A_{n-1} C_{n-1} B_{n-1}$ is $(10 \cdots 01)$. So the sum of these two vectors gives the same entries as the first row of $x_{n}^{2^{n}}$.

Now we want to check that the previous representations of the dihedral, quaternion and semidihedral groups are of minimal rank. To check this we will compute the exponent of a Sylow 2-subgroup of a general linear group over $\mathbb{F}_{2}$.

Lemma A.4. Let $M$ be an element of order $2^{n}$ in $\mathrm{GL}_{m}\left(\mathbb{F}_{2}\right)$. Then $2^{n-1}<m$. Moreover, there exists an element of order $2^{n}$ in the linear group $\mathrm{GL}_{2^{n-1}+1}\left(\mathbb{F}_{2}\right)$.

Proof. Let $M$ be an element of order $2^{n}$. Then $M$ satisfies the polynomial $X^{2^{n}}-1$. As we are in characteristic 2 we get $X^{2^{n}}-1=(X-1)^{2^{n}}$. The minimal polynomial of $M$ must be of the form $(X-1)^{r}$, and the characteristic polynomial must be $(X-1)^{m}$, with $r \leq m$. If $2^{n-1} \geq r$, then $M$ would satisfy the polynomial $(X-1)^{2^{n-1}}=$ $\left(X^{2^{n-1}}-1\right)$, so the order of $M$ would be at most $2^{n-1}$. So we get $2^{n-1}<r \leq m$.

Finally, the element $x_{n-1}$ in Lemma $\mathrm{A.3}$ is of order $2^{n}$ in $\mathrm{GL}_{2^{n-1}+1}\left(\mathbb{F}_{2}\right)$. 


\section{Appendix B. Projective Resolutions And the Yoneda Cocomplex}

B.1. Quaternion groups. We can find a projective resolution of $Q_{2^{n}}$ in CE56, p. 253], but before giving it we need some notation. Recall from equation (2) the notation for the generators and cohomology of the quaternion groups:

$$
Q_{2^{n}}=\left\langle x, z \mid x^{2^{n-1}}=1, z^{2}=x^{2^{n-2}}, z x z^{-1}=x^{-1}\right\rangle .
$$

Consider the following elements in the group algebra $\mathbb{F}_{2}\left[Q_{2^{n}}\right]$ :

$$
\begin{aligned}
& I=1+x, J=1+z, K=1+x z, L=1+x+x^{2}+\cdots+x^{2^{n-2}-1}, \\
& N_{x}=1+x+x^{2}+\cdots+x^{2^{n-1}-1} \text { and } N=\sum_{g \in Q_{2} n} g .
\end{aligned}
$$

Lemma B.1. Consider the elements $I, J, K, L, N_{x}$ and $N$ defined above. Then we have the following relations:

$$
\begin{aligned}
& L=I^{2^{n-2}-1}, N_{x}=I^{2^{n-1}-1}, I^{2^{n-2}}=J^{2}=K^{2}, I^{2^{n-1}}=J^{4}=K^{4}=0, \\
& K I=I J, K=I+J+I J \text { and } N=J N_{x}=N_{x} J=K N_{x}=N_{x} K .
\end{aligned}
$$

Proof. Recall that, as we are taking coefficients in $\mathbb{F}_{2},(1+u)^{2^{m}}=1+u^{2^{m}}$ and $(1+u)^{2^{m}-1}=1+u+\cdots+u^{2^{m}-1}$. So using this and the order of the elements $x, z$ and $x z$ in $Q_{2^{n}}$ we get all the equalities in the first row. The first two equalities of the second row can be computed directly. The last equality can be deduced from the fact that all elements in $Q_{2^{n}}$ can be written as $x^{i} z^{\varepsilon}$, for $0 \leq i \leq 2^{n-1}-1$ and $0 \leq \varepsilon \leq 1$ in just one way, so all elements appear as a summand in the products $J N_{x}, N_{x} J, K N_{x}$ and $N_{x} K$.

The following result can be found in CE56, p. 253], and we include it here to formulate it using the elements in equation (10):

Lemma B.2. A projective resolution of $\mathbb{F}_{2}$ as an $\mathbb{F}_{2}\left[Q_{2^{n}}\right]$-module is given by the following periodic data:

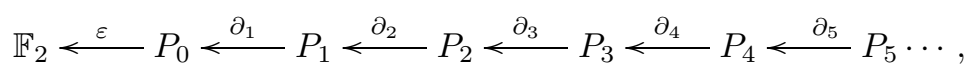

where $P_{4 i} \cong P_{4 i+3} \cong \mathbb{F}_{2}\left[Q_{2^{n}}\right]$ and $P_{4 i+1} \cong P_{4 i+2} \cong \mathbb{F}_{2}\left[Q_{2^{n}}\right]^{2}\left(=\mathbb{F}_{2}\left[Q_{2^{n}}\right] \oplus \mathbb{F}_{2}\left[Q_{2^{n}}\right]\right)$ and the differentials: $\partial_{4 i+1}=\left(\begin{array}{ll}I & J\end{array}\right), \partial_{4 i+2}=\left(\begin{array}{cc}L & K \\ I\end{array}\right), \partial_{4 i+3}=\left(\begin{array}{c}I \\ K\end{array}\right)$ and $\partial_{4 i}=(N)$.

Now we want to express the generators in the cohomology of quaternion groups as a cochain maps in the Yoneda cocomplex. We refer the interested reader to Bor01] for the use of the Yoneda cocomplex in group cohomology.

Recall the cohomology of the quaternion groups from equation (3):

$$
\begin{aligned}
& H^{*}\left(B Q_{8}\right) \cong \mathbb{F}_{2}[X, Y, V] /\left(X^{2}+X Y+Y^{2}, X^{2} Y+X Y^{2}\right) \text { and } \\
& H^{*}\left(B Q_{2^{n}}\right) \cong \mathbb{F}_{2}[X, Y, V] /\left(X^{2}+X Y, Y^{3}\right) \text { for } n \geq 4
\end{aligned}
$$

with $\operatorname{deg}(X)=\operatorname{deg}(Y)=1$ and $\operatorname{deg}(V)=4$.

Lemma B.3. The generators $X, Y$ and $V$ in $H^{*}\left(B Q_{2^{n}}\right)$ can be represented in the Yoneda cocomplex by the following cochain maps:

- The generator $X$ as the cochain map $X_{i}: P_{i+1} \rightarrow P_{i}$ defined as (to cover the case $Q_{8}$ here we are using the convention $I^{0}=1$ ):

$$
\begin{aligned}
& X_{4 i}=(10), X_{4 i+1}=\left(\begin{array}{cc}
I^{2^{n-2}-2} & 1 \\
0 & 1+I
\end{array}\right), \\
& X_{4 i+2}=\left(\begin{array}{l}
1 \\
1
\end{array}\right) \text { and } X_{4 i+3}=\left(I^{2^{n-1}-2} J\right) .
\end{aligned}
$$


- The element $Y$ in $H^{1}\left(B Q_{2^{n}}\right)$ by a cochain map $Y_{i}: P_{i+1} \rightarrow P_{i}$ defined as:

$$
Y_{4 i}=\left(\begin{array}{ll}
0 & 1
\end{array}\right), Y_{4 i+1}=\left(\begin{array}{ll}
0 & 1 \\
1 & 0
\end{array}\right), Y_{4 i+2}=\left(\begin{array}{l}
0 \\
1
\end{array}\right) \text { and } Y_{4 i+3}=\left(N_{x}\right) \text {. }
$$

- Finally the element $V \in H^{4}\left(B Q_{2^{n}}\right)$ by a cochain map defined by $V_{i}: P_{i+4} \rightarrow$ $P_{i}$ which is the identity.

Proof. We have that the projective resolution from Lemma B.2 is minimal, in the sense that $P_{i} \cong \mathbb{F}_{2}\left[Q_{2^{n}}\right]^{\operatorname{dim}\left(H^{i}\left(Q_{2^{n}}\right)\right)}$, so all $\mathbb{F}_{2}\left[Q_{2^{n}}\right]$-morphisms $E: P_{1} \rightarrow \mathbb{F}_{2}$ must lift to maps $E_{i}$ to give the following commutative diagram:

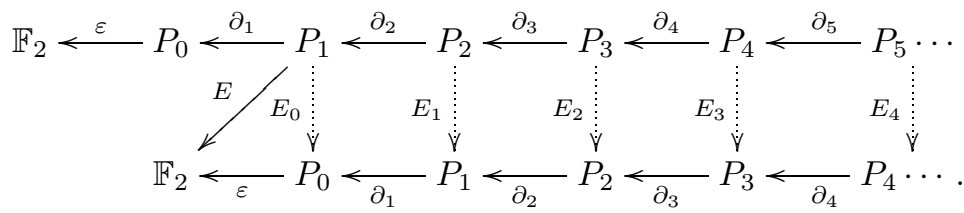

As $P_{1}=\mathbb{F}_{2}\left[Q_{2^{n}}\right]^{2}$ we can start looking for the lifting of the $\mathbb{F}_{2}\left[Q_{2^{n}}\right]$-linear map $\tilde{X} \stackrel{\text { def }}{=}\left(\begin{array}{ll}1 & 0\end{array}\right)$ (respectively $\tilde{Y} \stackrel{\text { def }}{=}\left(\begin{array}{ll}0 & 1\end{array}\right)$ ). Observe that the map $X_{j}: P_{j+1} \rightarrow P_{j}$ (respectively $Y_{j}: P_{j+1} \rightarrow P_{j}$ ) defined in the statement is indeed a lifting for $\tilde{X}$ (respectively $\tilde{Y}$ ). This fact can be checked by multiplying the matrices $\partial_{\bullet}$ defined in Lemma B.2 and $X_{\bullet}$ (respectively $Y_{\bullet}$ ) defined in the statement. To get the equality of both sides one has to apply the relations given in Lemma B.1.

As elements in $H^{*}\left(Q_{2^{n}}\right)$ we can compute the multiplication involving $\tilde{X}$ and $\tilde{Y}$ composing the cochain maps. Doing that we will get that $\tilde{X}^{2}+\tilde{X} \tilde{Y}=0$ and $\tilde{Y}^{3}=0$, obtaining that $\tilde{X}$ (respectively $\tilde{Y}$ ) can be taken as a representative of $X$ in the Yoneda cocomplex (respectively $Y$ ).

Finally a nonzero element in $H^{4}\left(Q_{2^{n}}\right)$ which will represent $V$ is a lifting of (1): $P_{4} \rightarrow \mathbb{F}_{2}$, and, as $P_{i+4} \cong P_{i}$ and the differential are also periodic, we can take the identity.

B.2. Semidihedral groups. In this subsection we will proceed in a similar way as in the previous one. The main difference with the quaternion family comes from the fact that, for semidihedral groups, there is not such a nice projective resolution.

Recall from equation (2) a finite presentation for the semidihedral groups:

$$
S D_{2^{n}}=\left\langle x, t \mid x^{2^{n-1}}=1, t^{2}=1, t x t^{-1}=x^{2^{n-2}-1}\right\rangle .
$$

Consider the following elements in the group algebra $\mathbb{F}_{2}\left[S D_{2^{n}}\right]$ :

$$
\begin{aligned}
& I=1+x, J=1+t, L=1+x+x^{2}+\cdots+x^{2^{n-2}-2} \text { and } \\
& N_{x}=1+x+x^{2}+\cdots+x^{2^{n-1}-1} .
\end{aligned}
$$

The following equalities will be used in the computation of the generators in the Yoneda cocomplex and their products.

Lemma B.4. Consider the elements $I, J, L$ and $N_{x}$ defined above. Then we have the following relations:
(a) $L=I^{2^{n-2}-1}+x^{2^{n-2}-1}, I^{2^{n-1}}=J^{2}=0$,
(b) $(1+t L) I=I J,\left(1+t L^{n}\right) I=I\left(1+t L^{n-1}\right)$,
(c) $t N_{x}=N_{x} t, t I^{2^{n-1}-2}=I^{2^{n-1}-2} t$,
(d) $I^{2^{n-2}-2} t=t I^{2^{n-2}-2}$,
(e) $\left(1+t L^{2 i}\right)^{2}=0,\left(1+t L^{2 i+1}\right)^{2}=N_{x}$ and $L^{2^{n-1}}=1$. 
Proof. (a) One proceeds as in the proof of the first part of Lemma B.1.

(b) This can be done directly. As an example we do the first equality: $I J=$ $(1+x)(1+t)=1+x+t+x t=1+x+t+t x^{2^{n-2}-1}$ and $(1+t L) I=$ $\left(1+t+t x+\cdots t x^{2^{n-2}-2}\right)(1+x)=1+x+t+t x^{2^{n-2}-1}$, where in the last equality we are using that when we expand the product most of the summands cancel in $\mathbb{F}_{2}$.

(c) In the first equality, we use the commutation rules between $t$ and $x$ in the presentation of $S D_{2^{n}}$. In the second the fact that $I^{2^{n-1}-2}$ is the sum of all possible even powers of $x$ (including $x^{0}=1$ ) and the commutation rules between $t$ and $x$ preserve the exponent of $x$ modulo 2 .

(d) The element $I^{2^{n-2}-2}$ is the sum of all even powers of $x$ up to degree $2^{n-2}-2$. The commutation rule between $x$ and $t$ adds $2^{n-2}$ to the exponent of $x$ and changes the sign, so at the end we have the same exponents.

(e) The first two equalities use the equality, in characteristic $2,(1+u)^{2}=1+u^{2}$ in each case, and apply the commutation rules between $x$ and $t$ to get the desired result. The last statement also follows from the same equality in $\mathbb{F}_{2}$ : $L^{2^{n-1}}=1+x^{2^{n-1}}+\left(x^{2}\right)^{2^{n-1}}+\cdots+\left(x^{2^{n-2}}\right)^{2^{n-1}}$ and as $\left(x^{i}\right)^{2^{n-1}}=1$ we get the sum of an odd number of 1 s, so $L^{2^{n-1}}=1$.

We can find a projective resolution for this family of groups in Wal61:

Lemma B.5. A projective resolution of $\mathbb{F}_{2}$ as an $\mathbb{F}_{2}\left[S D_{2^{n}}\right]$-module is given by:

$$
\mathbb{F}_{2} \longleftarrow \varepsilon P_{0} \longleftarrow \partial_{1} P_{1} \longleftarrow \partial_{2} P_{2} \longleftarrow \partial_{3} P_{3} \stackrel{\partial_{4}}{\longleftarrow} P_{4} \longleftarrow \partial_{5} P_{5} \cdots,
$$

where $P_{i} \cong \mathbb{F}_{2}\left[S D_{2^{n}}\right]^{i+1}\left(=\mathbb{F}_{2}\left[S D_{2^{n}}\right] \oplus \stackrel{i+1}{\text {. }} \oplus \mathbb{F}_{2}\left[S D_{2^{n}}\right]\right)$ and the differentials are defined inductively: $\partial_{1}=\left(\begin{array}{ll}I & J\end{array}\right)$,

$$
\begin{gathered}
\partial_{2 i}=\left(\begin{array}{c|cccc}
N_{x} & 1+t L^{i} & 0 & \cdots & 0 \\
\hline 0 & & & & \\
\vdots & & \partial_{2 i-1} & & \\
0 & & & &
\end{array}\right) \text { and } \\
\partial_{2 i+1}=\left(\begin{array}{c|ccccc}
I & 1+t L^{i} & i & 0 & \cdots & 0 \\
\hline 0 & & & & \\
\vdots & & \partial_{2 i} & & \\
0 & & &
\end{array}\right),
\end{gathered}
$$

where $I, J, L$ and $N_{x}$ are the elements defined in equation (11).

Now we need the cohomology of $S D_{2^{n}}$, which has been introduced in equation (3):

$$
H^{*}\left(B S D_{2^{n}}\right) \cong \mathbb{F}_{2}[X, Y, U, V] /\left(X^{2}+X Y, X U, X^{3}, U^{2}+\left(X^{2}+Y^{2}\right) V\right),
$$

with $\operatorname{deg}(X)=\operatorname{deg}(Y)=1, \operatorname{deg}(U)=3$ and $\operatorname{deg}(V)=4$.

We now proceed to give cochain maps representing the generators. This free resolution does not allow us to give inductive formulas, but as we are just interested in 4-fold iterated Massey products of elements in degrees 1 and 2 we just need to give the first four steps of the maps. 
Before giving the element we fix the following basis of this cohomology as a graded vector space in low degrees: $\{X, Y\}$ in degree one, $\left\{X^{2}, Y^{2}\right\}$ in degree two, $\left\{U, Y^{3}\right\}$ in degree three and $\left\{Y^{4}, Y U, V\right\}$ in degree four.

Lemma B.6. The representing cochain maps in the Yoneda cocomplex of the generators $X, Y, U$ and $V$ in $H^{*}\left(B S D_{2^{n}}\right)$ are characterized by the following data:

- The element $X \in H^{1}\left(B S D_{2^{n}}\right)$ can be taken as a cochain morphism $X_{i}: P_{i+1}$ $\rightarrow P_{i}$ with

$$
\begin{aligned}
& X_{0}=\left(\begin{array}{ll}
1 & 0
\end{array}\right), \quad X_{1}=\left(\begin{array}{c|cc}
I^{2^{n-1}-2} & t\left(L+I^{2^{n-2}-2}\right) & 0 \\
\hline 0 & X_{0}
\end{array}\right), \\
& X_{2}=\left(\begin{array}{c|ccc}
1 & t\left(L+I^{2^{n-2}-2}\right) & 1 & 0 \\
\hline 0 & X_{1} &
\end{array}\right) \quad \text { and } \\
& X_{3}=\left(\begin{array}{c|cccc}
I^{2^{n-1}-2} & 0 & 0 & 0 & 0 \\
\hline 0 & & & & \\
0 & & X_{2} & \\
0 & & & &
\end{array}\right) .
\end{aligned}
$$

- The element $Y \in H^{1}\left(B S D_{2^{n}}\right)$ can be taken as a cochain map $Y_{i}: P_{i+1} \rightarrow P_{i}$ with $Y_{i}=\left(0 \mid \operatorname{Id}_{i+1}\right)$, where $\operatorname{Id}_{i+1}$ is the $(i+1) \times(i+1)$ identity matrix.

- Either $U$ or $U+Y^{3}$ is represented by a lifting of the map from $P^{3}$ to $\mathbb{F}_{2}$ given by the matrix (1000).

- Either $V$ or $Y U$ can be taken as a lifting of the map from $P^{4}$ to $\mathbb{F}_{2}$ given by the matrix (10000).

Proof. Here we give all the arguments which are used in the proof, but, for the sake of clarity, avoiding explicit computations. The computations we avoid can be checked directly using the relations in Lemma B.4.

The elements in $H^{1}\left(B S D_{2^{n}}\right)$ are determined by $\mathbb{F}_{2}\left[Q_{2^{n}}\right]$-morphisms $P_{1} \rightarrow \mathbb{F}_{2}$. As $P_{1}=\mathbb{F}_{2}\left[S D_{2^{n}}\right]^{2}=\operatorname{dim}\left(H^{1}\left(B S D_{2^{n}}\right)\right.$, both morphisms given by matrices $(10)$ and $\left(\begin{array}{ll}0 & 1\end{array}\right)$ must lift. The statement in the lemma tells us which one is the lifting for each map $\left(X_{i}\right.$ and $Y_{i}$ respectively). Now we can decide which one is $X$ and $Y$ in $H^{*}\left(B S D_{2^{n}}\right)$ checking, for example, the relation $X^{3}=0$.

The elements in $H^{3}\left(B S D_{2^{n}}\right)$ are determined by $\mathbb{F}_{2}\left[Q_{2^{n}}\right]$-morphisms $P_{3} \rightarrow \mathbb{F}_{2}$ which can be lifted to cochain maps, and we can write them as matrices $1 \times 4$. Using the previous representatives, and $Y^{3}$ is determined by $\left(\begin{array}{lll}0 & 0 & 0\end{array}\right), X^{3}=X^{2} Y=X Y^{2}$ (which is a coboundary) is represented by $(0010)$. Observe that $(0100)$ cannot be lifted, so it does not represent any element in $H^{3}\left(B S D_{2^{n}}\right)$. So it remains $\left(\begin{array}{llll}1 & 0 & 0 & 0\end{array}\right)$, which can be lifted and, taking into account the dimension of the cohomology in degree 3 , it can be considered as a representative for $U$ or $U+Y^{3}$.

The same arguments work for detecting which can be $V$ as a $\mathbb{F}_{2}\left[Q_{2^{n}}\right]$-morphism

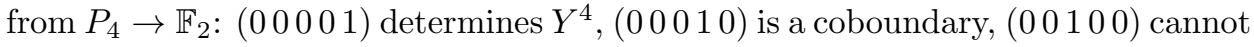
be lifted, $\left(\begin{array}{lllll}0 & 1 & 0 & 0 & 0\end{array}\right)$ determines $Y U$, so $V($ or $V+Y U)$ can be taken as $\left(\begin{array}{lllll}1 & 0 & 0 & 0 & 0\end{array}\right)$.

\section{REFERENCES}

[Bag92] Czesław Bagiński, Modular group algebras of 2-groups of maximal class, Comm. Algebra 20 (1992), no. 5, 1229-1241. MR1157906 (93a:20010) 
[Ben92] D. J. Benson, Resolutions and Poincaré duality for finite groups, Algebraic topology (San Feliu de Guíxols, 1990), Lecture Notes in Math., vol. 1509, Springer, Berlin, 1992, pp. 10-19. MR.1185957 (93i:20057)

[BK72] A. K. Bousfield and D. M. Kan, Homotopy limits, completions and localizations, Lecture Notes in Mathematics, Vol. 304, Springer-Verlag, Berlin, 1972. MR0365573 (51:1825)

[BK07] Czesław Bagiński and Alexander Konovalov, The modular isomorphism problem for finite p-groups with a cyclic subgroup of index $p^{2}$, Groups St. Andrews 2005. Vol. 1, London Math. Soc. Lecture Note Ser., vol. 339, Cambridge Univ. Press, Cambridge, 2007, pp. 186-193. MR2327323 (2008f:20009)

[BL97] Carles Broto and Ran Levi, On the homotopy type of BG for certain finite 2-groups $G$, Trans. Amer. Math. Soc. 349 (1997), no. 4, 1487-1502. MR.1370636 (97h:55016)

[BL02] Carles Broto and Ran Levi, On spaces of self-homotopy equivalences of p-completed classifying spaces of finite groups and homotopy group extensions, Topology 41 (2002), no. 2, 229-255. MR1876889 (2002j:55013)

[Bor01] Inger Christin Borge, A cohomological approach to the classification of p-groups, Ph.D. thesis, Oxford University, 2001.

[Bor04] _ A cohomological approach to the modular isomorphism problem, J. Pure Appl. Algebra 189 (2004), no. 1-3, 7-25. MR2038560 (2005c:20006)

[Car77] Jon F. Carlson, Periodic modules over modular group algebras, J. London Math. Soc. (2) 15 (1977), no. 3, 431-436. MR0472985 (57:12664)

[CE56] Henri Cartan and Samuel Eilenberg, Homological algebra, Princeton University Press, Princeton, N. J., 1956. MR0077480 (17:1040e)

[Dwy75] William G. Dwyer, Homology, Massey products and maps between groups, J. Pure Appl. Algebra 6 (1975), no. 2, 177-190. MR0385851 (52:6710)

[Kra66] David Kraines, Massey higher products, Trans. Amer. Math. Soc. 124 (1966), 431-449. MR0202136(34:2010)

[May69] J. Peter May, Matric Massey products, J. Algebra 12 (1969), 533-568. MR0238929 $(39: 289)$

[Wal61] C. T. C. Wall, Resolutions for extensions of groups, Proc. Cambridge Philos. Soc. 57 (1961), 251-255. MR0178046 (31:2304)

Departament de Matemàtiques, Universitat Autònoma de Barcelona, 08193 CerdanyOLA DEL VALlÈs, SPAin

E-mail address: Albert.Ruiz@uab.cat

Departamento de Álgebra, Geometría y Topología, Universidad de Málaga, Apdo CORReos 59, 29080 MÁlaga, Spain

E-mail address: viruel@agt.cie.uma.es 